\title{
Asymptotic Models for the Electric Potential across a Highly Conductive Casing
}

\author{
A. ERDOZAIN $N^{\mathrm{a}, \mathrm{b}}$, V. PÉRON ${ }^{\mathrm{a}, \mathrm{b}, \mathrm{d}}$, D. PARDO ${ }^{\mathrm{c}}$ \\ ${ }^{a}$ Team MAGIQUE-3D (INRIA Bordeaux - Sud-Ouest) \\ ${ }^{b}$ Laboratoire de Mathématiques et de leurs Applications de Pau (LMA-PAU) \\ ${ }^{c}$ Ikerbasque, University of the Basque Country (UPV/EHU), and Basque Center for Applied Mathematics (BCAM) \\ ${ }^{d}$ Université de Pau et des Pays de l'Adour
}

\begin{abstract}
We analyze a configuration that involves a steel-cased borehole, where the casing that covers the borehole is considered as a highly conductive thin layer. We develop an asymptotic method for deriving reduced problems capable of efficiently dealing with the numerical difficulties caused by the casing when applying traditional numerical methods. We derive several reduced models by employing two different approaches, each of them leading to different classes of models. We prove stability and convergence results for these models. The theoretical orders of convergence are supported by numerical results obtained with the finite element method.
\end{abstract}

Keywords: asymptotic models, impedance conditions, electric potential, thin layer, finite element method, borehole

\section{Introduction}

Borehole resistivity measurements are a common technique employed for characterizing the Earth's subsurface. The standard procedure for acquiring resistivity measurements consists in employing electromagnetic waves. A logging instrument equipped with a transmitter and several receivers is placed inside a borehole and electromagnetic waves are sent to the surrounding layered formation. Then, the waves are measured at the receivers and this information is employed to determine the resistivity of the formation.

Electrical logging through casing is of special interest in wells surrounded by a steel-made casing. While such casing protects the well from a possible collapse, at the same time it greatly complicates the numerical simulations due to the extreme thinness of the casing, along with the high conductivity it presents. Thus, when dealing with this kind of configurations, the numerical results delivered by standard techniques are often inaccurate or simply too costly to be performed in real time.

According to the results shown in $[15,16]$, the second derivative of the electric potential along the vertical direction can be employed to approximate the conductivity of the surrounding layered formation. This technique has been widely used in the literature for acquiring and interpreting borehole through-casing resistivity measurements. See, for instance, [18, 19, 20, 17, 21, 22, 5, 4].

These studies are commonly approached from two different perspectives: numerical methods and semi-analytical ones. While semi-analytical methods provide very efficient solutions, they are severely limited when considering realistic configurations. Numerical methods, on the other hand, are capable of dealing with complex realistic configurations. Hence, they seem a suitable option for dealing with these kind of scenarios. However, the use of numerical methods becomes challenging when a casing surrounds the borehole, due to its high electrical conductivity and its small thickness. These features lead to a dramatic increase of the computational cost, and traditional numerical methods do not perform well. Thus, it is relevant to develop techniques to avoid the problems caused by the metallic casing and to construct reduced problems involving appropriate boundary or transmission conditions.

In this study, we develop an asymptotic method to solve a problem involving a realistic configuration [22], where the conductivity in the casing takes much higher values than those in the layered formation. In this configuration, the

Email address: victor.peron@univ-pau.fr(V. PÉRON) 
casing can be seen as a thin layer of uniform thickness $\varepsilon$ and its conductivity $\sigma$ is proportional to the third negative power of $\varepsilon$, i.e., $\sigma=\widehat{\sigma}_{0} \varepsilon^{-3}$. Such choice is motivated by the fact that a typical steel casing used in borehole applications is approximately $1.27 \mathrm{~cm}$ thick and exhibits a conductivity of $5 \times 10^{5} \mathrm{~S} / \mathrm{m}$. Notice, however, that our approach can also be applied for values of $\widehat{\sigma}_{0}$ different from (although close to) one, which covers almost all existing borehole steel casings. For a given casing, its thickness and conductivity are constant, so we simply write $\widehat{\sigma}_{0}$ rather than $\widehat{\sigma}(\varepsilon)$.

Our aim is to derive Impedance Transmission Conditions (ITCs) for the electric potential across the aforementioned casing. The naturally small thickness of the casing compared to the rest of the domain makes it ideal for applying this kind of method. Such conditions are derived by performing an asymptotic expansion of the solution and are specifically designed to use them in replacement of one part of the computational domain (in our case, the subdomain occupied by the casing). The concept of ITCs is rather classical in the modeling of electromagnetic wave propagation phenomena. See, for instance, [7, 13, 12, 9, 6, 25, 28, 29, 23], where ITCs are derived to substitute a thin layer present in the configuration.

In this work, we consider high-contrast material properties. This greatly increases the complexity of the problem. Several works can be found with similarities in this matter, for example, [26, 27, 13] perform studies in electromagnetism where the material properties depend on the thickness of a thin layer. However, the derivation of ITCs does not only concern the field of electromagnetism. In [3, 24, 11, 10, 2], we find asymptotic studies related with the fields of Elasticity and Acoustics.

Here, we consider a transmission problem for the static electric potential set in a cylindrically shaped axisymmetric domain $\Omega \subset \mathbb{R}^{3}$, which is complemented with homogeneous Dirichlet boundary conditions. This domain is composed of three subdomains: $\Omega_{\text {int }}^{\varepsilon}$, which represents the interior of the borehole; $\Omega_{\mathrm{ext}}^{\varepsilon}$, corresponding to the rock formation; and $\Omega_{\text {lay }}^{\varepsilon}$, which constitutes the casing and it consists of a thin layer of uniform thickness $\varepsilon$. In this framework, we derive ITCs for the electric potential employing two different approaches. The first one consists of deriving the transmission conditions across the thin layer, obtaining thus models set in $\Omega_{\text {int }}^{\varepsilon}$ and $\Omega_{\mathrm{ext}}^{\varepsilon}$. We shall refer to this class of ITCs as Gap-ITCs. The second approach tackles the problem by deriving the transmission conditions across an artificial interface situated in the middle of the thin layer, resulting thus in models with no gap in the middle. We shall refer to these class of ITCs as Interface-ITCs. Both classes have their advantages and drawbacks, which are discussed in this work. In particular, interface-ITCs do not provide any savings in terms of the number of degrees of freedom; however, they provide an alternative treatment to the problem, which may exhibit some advantages. For example, since we only work with two materials and one interface condition in this case, perhaps it would be possible to more easily obtain analytical solutions than with other approaches, and/or employ an efficient numerical mode-matching method to solve the problem.

The proposed asymptotic method we develop consists of the following steps: First, we scale the subdomain occupied by the thin layer. Then, we select an Ansatz for the electric potential in the form of power series of $\varepsilon$ and we obtain a collection of problems that can be alternately solved to determine the elementary problems satisfied by each term of the asymptotic expansion. After that, we truncate the resulting series and collect the first terms of the expansion to infer equivalent conditions by neglecting residual terms depending on $\varepsilon$. Finally, we prove convergence results for the derived asymptotic models.

This paper follows the next outline: In Section 2, we introduce the model problem, along with all the elements that compose the configuration. Section 3 is devoted to the main contributions, including asymptotic models with Gap-ITCs and Interface-ITCs. Then, several numerical results are shown in Section 4. Here, numerical results for validating the convergence rates of the asymptotic models are presented, and then, an application to the field of resistivity measurements is carried out. Finally, two appendices are included. In Appendix A, we detail every step of the asymptotic method employed in the derivation of the asymptotic models. In Appendix B, we present stability and convergence results for the derived asymptotic models.

We denote by $H^{s}(\Omega)$ the standard Sobolev space, endowed with its natural norm $\|\cdot\|_{s, \Omega}$.

\section{The model problem}

Let $\Omega \subset \mathbb{R}^{3}$ be the domain of interest depicted in Figure 1. Domain $\Omega$ is cylinder shaped and can be decomposed into three subdomains: $\Omega_{\text {int }}^{\varepsilon}, \Omega_{\text {ext }}^{\varepsilon}$, and $\Omega_{\text {lay }}^{\varepsilon}$, where subdomain $\Omega_{\text {lay }}^{\varepsilon}$ is a thin layer of uniform thickness $\varepsilon>0$. These 
subdomains can be described as follows:

$$
\begin{aligned}
& \Omega_{\text {int }}^{\varepsilon}=\left\{(r, \theta, z) \in \mathbb{R}^{3}: 0 \leq r<r_{0}-\frac{\varepsilon}{2}, 0 \leq \theta<2 \pi, z_{0}<z<z_{1}\right\}, \\
& \Omega_{\text {lay }}^{\varepsilon}=\left\{(r, \theta, z) \in \mathbb{R}^{3}: r_{0}-\frac{\varepsilon}{2}<r<r_{0}+\frac{\varepsilon}{2}, 0 \leq \theta<2 \pi, z_{0}<z<z_{1}\right\}, \\
& \Omega_{\text {ext }}^{\varepsilon}=\left\{(r, \theta, z) \in \mathbb{R}^{3}: r_{0}+\frac{\varepsilon}{2}<r<R_{0}, 0 \leq \theta<2 \pi, z_{0}<z<z_{1}\right\} .
\end{aligned}
$$

We denote by $\Gamma_{\text {int }}^{\varepsilon}$ the interface between $\Omega_{\text {int }}^{\varepsilon}$ and $\Omega_{\text {lay }}^{\varepsilon}$, and by $\Gamma_{\text {ext }}^{\varepsilon}$ the interface between $\Omega_{\text {lay }}^{\varepsilon}$ and $\Omega_{\text {ext }}^{\varepsilon}$. In this domain, we study the equation for the static electric potential, complemented with homogeneous Dirichlet boundary conditions. The equations read as follows:

$$
\left\{\begin{aligned}
\operatorname{div}(\sigma \nabla u)=f & \text { in } \quad \Omega \\
u=0 & \text { on } \quad \partial \Omega .
\end{aligned}\right.
$$

Here, $u$ represents the electric potential, $\sigma$ stands for the conductivity and $f$ is the right-hand side, which corresponds

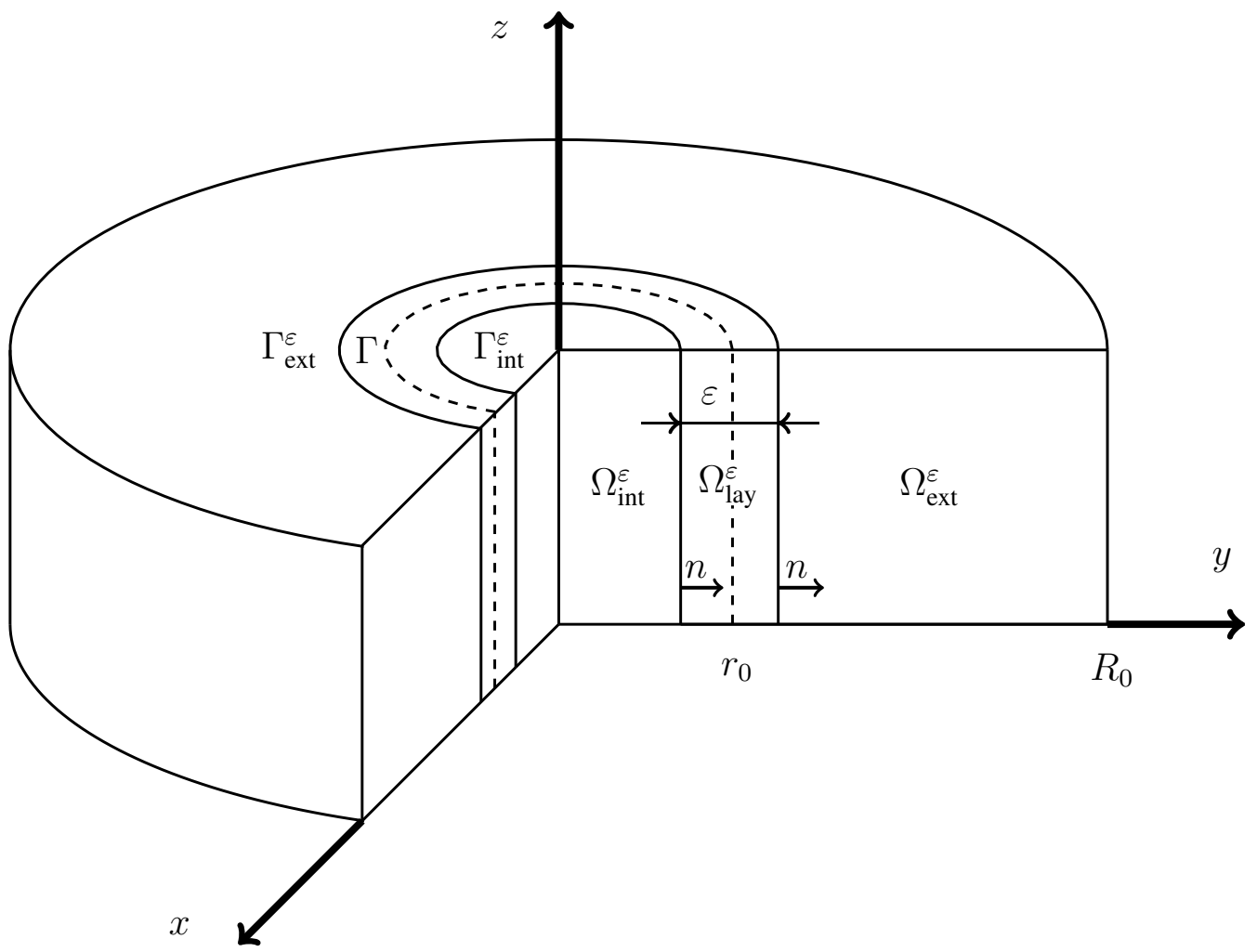

Figure 1: Sectioned three dimensional domain for the model problem and asymptotic models with Gap-ITCs.

to a current source. The conductivity is a piecewise constant scalar-valued function with different values in each subdomain. Specifically, the value of the conductivity inside the thin layer $\Omega_{\text {lay }}^{\varepsilon}$ is much larger than the one in the other subdomains, and we express it as a function of parameter $\varepsilon$. We also assume the right-hand side $f$ is a piecewise smooth function, independent of $\varepsilon$, which vanishes inside the layer. The conductivity and the right-hand side take the 
following form:

$$
\sigma=\left\{\begin{array}{ll}
\sigma_{\text {int }} & \text { in } \Omega_{\text {int }}^{\varepsilon}, \\
\sigma_{\text {lay }}=\widehat{\sigma}_{0} \varepsilon^{-3} & \text { in } \Omega_{\text {lay }}^{\varepsilon}, \\
\sigma_{\text {ext }} & \text { in } \Omega_{\text {ext }}^{\varepsilon},
\end{array} \quad f= \begin{cases}f_{\text {int }} & \text { in } \Omega_{\text {int }}^{\varepsilon}, \\
f_{\text {lay }}=0 & \text { in } \Omega_{\text {lay }}^{\varepsilon}, \\
f_{\text {ext }} & \text { in } \Omega_{\text {ext }}^{\varepsilon},\end{cases}\right.
$$

where $\sigma_{\text {int }}, \sigma_{\text {ext }}, \widehat{\sigma}_{0}>0$ are given constants.

Since the interface problem (2) is elliptic, its solution $u$ has an optimal piecewise regularity depending on both the regularity of the domain and the regularity of the data $f$. For general data $f \in L^{2}(\Omega)$, the solution $u$ belongs to $P H^{2}(\Omega)$ (i.e. piecewise $H^{2}$ ). For piecewise smooth function $f$, the solution $u$ belongs to $P H^{3-\delta}(\Omega)$ for any $\delta>0$ (here the bound on the Sobolev exponent comes from the edge singularities on the boundary of the domain, see for instance $[8,23]$ ) ; the solution $u$ belongs to piecewise $\mathcal{C}^{1}(\bar{\Omega})$ functions thanks to Sobolev embeddings in three dimensions. Hence, there is no 'singular' behavior appearing in the vicinity of the top and the bottom of the cylinder.

\section{Main Results. Asymptotic models}

We apply an asymptotic method for obtaining equivalent models for Problem (2). The method is described in detail in Appendix A, and consists of the following steps: First, we scale the domain corresponding to the thin layer by employing its thickness $\varepsilon$ (see Section A.1). Then, we select an Ansatz in the form of power series of $\varepsilon$ and we substitute it in the equations of Problem (2) (see Section A.2). By grouping the terms that share the same power in $\varepsilon$, we obtain a collection of problems that can be alternately solved to determine the elementary problems satisfied by every term of the asymptotic expansion (see Section A.3). Finally, we truncate the resulting series and collect the first terms of the expansion to infer equivalent conditions by neglecting residual terms depending on $\varepsilon$ (see Sections A.4 and A.5).

Definition 1. Let $u^{[k]}$ be the solution to an asymptotic model defined over a domain $\Omega^{0} \subseteq \Omega$. Let $u$ be the solution to the reference problem. We say that the asymptotic model is of order $k+1$ if there exists a constant $C>0$ independent of $\varepsilon$, such that the following expression is satisfied for a sufficiently small $\varepsilon$ :

$$
\left\|u-u^{[k]}\right\|_{1, \Omega^{0}} \leq C \varepsilon^{k+1} .
$$

This method leads us to two different classes of transmission conditions: Gap-ITCs and Interface-ITCs. There is a main difference between the derivation of Interface-ITCs and the derivation of Gap-ITCs since we apply a formal Taylor series expansion to the terms of the asymptotic expansion to derive Interface-ITCs (see Section A.6). This way, we extend the domain of definition for these terms, considering thus domain $\Omega$, decomposed into subdomains $\Omega_{\text {int }}$ and $\Omega_{\mathrm{ext}}$, as depicted in Figure 2. Asymptotic models with Interface-ITCs are defined over these subdomains, which are described as follows:

$$
\begin{aligned}
& \Omega_{\text {int }}=\left\{(r, \theta, z) \in \mathbb{R}^{3}: 0 \leq r<r_{0}, 0 \leq \theta<2 \pi, z_{0}<z<z_{1}\right\}, \\
& \Omega_{\mathrm{ext}}=\left\{(r, \theta, z) \in \mathbb{R}^{3}: r_{0}<r<R_{0}, 0 \leq \theta<2 \pi, z_{0}<z<z_{1}\right\} .
\end{aligned}
$$

On the other hand, asymptotic models with Gap-ITCs are defined over the domain $\Omega^{\varepsilon}=\Omega_{\mathrm{int}}^{\varepsilon} \cup \Omega_{\mathrm{ext}}^{\varepsilon}$, where subdomains $\Omega_{\text {int }}^{\varepsilon}$ and $\Omega_{\mathrm{ext}}^{\varepsilon}$ are described by (1).

Further details concerning the process of derivation of the asymptotic models for both classes are available in Appendix A. The resulting asymptotic models converge towards the reference Model (2) with a certain order. Details concerning the proofs for the stability and convergence of these models are found in Appendix B.

It is possible to adapt the asymptotic method developed in Appendix A when the domain $\Omega$ is a cylindrical domain with arbitrary smooth-shaped base. It requires to use local coordinates along the interface $\Gamma$. In this framework, the resulting first order asymptotic models still coincide with the models presented in Section 3.1 and in Section 3.2. 


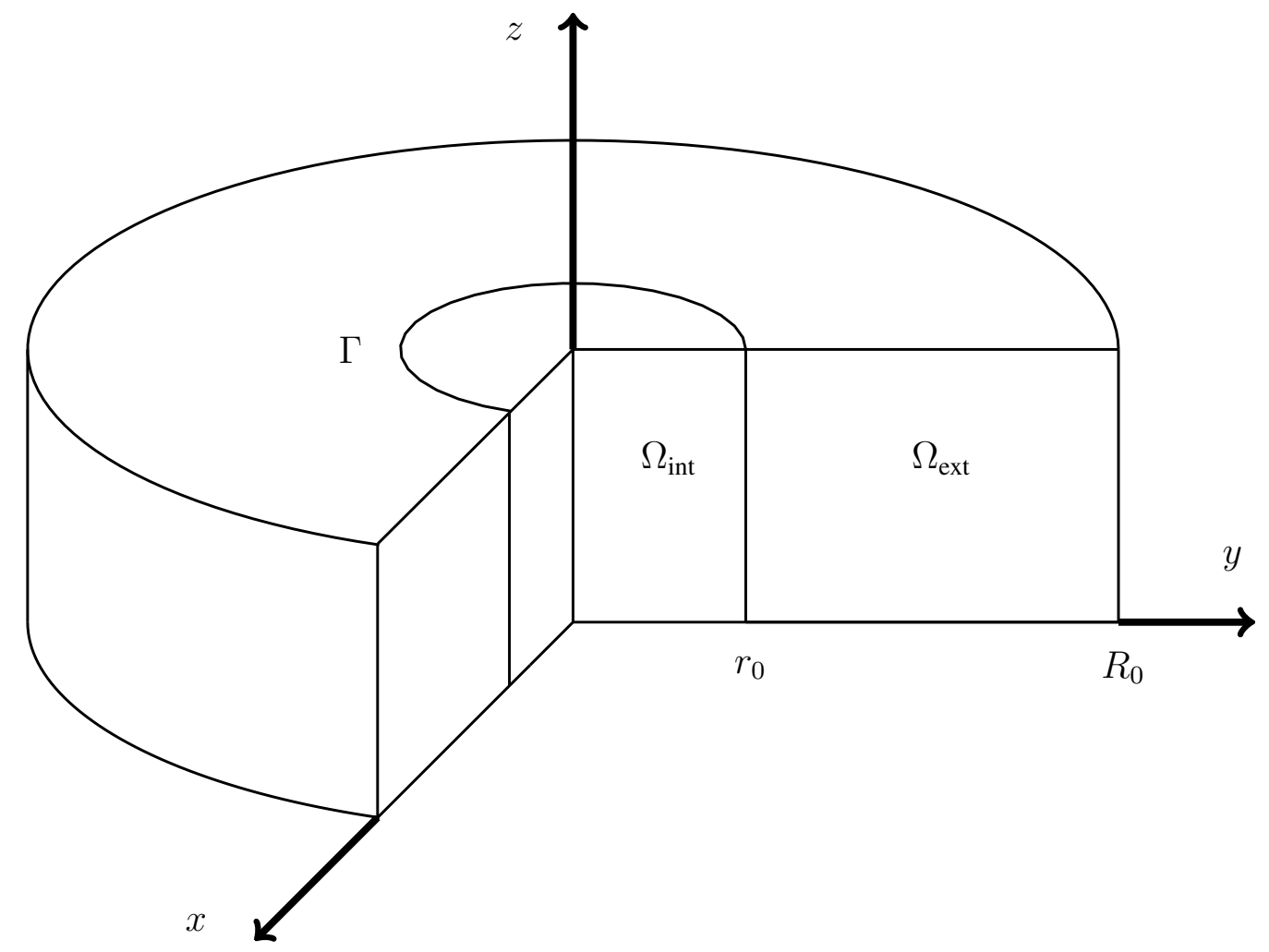

Figure 2: Sectioned domain for the asymptotic models with Interface-ITCs. 


\subsection{Asymptotic models with Gap-ITCs}

We apply the aforementioned method to derive approximate models of second and fourth order, respectively.

Definition 2. Let u be a smooth function defined over $\Omega^{\varepsilon}$. We define its jump and mean value across a thin layer $\Omega_{\text {lay }}^{\varepsilon}$ by:

$$
\left\{\begin{array}{c}
{[u]_{\Gamma^{\varepsilon}}=\left.u_{e x t}\right|_{\Gamma_{e x t}^{\varepsilon}}-\left.u_{i n t}\right|_{\Gamma_{i n t}^{\varepsilon}},} \\
\{u\}_{\Gamma^{\varepsilon}}=\frac{1}{2}\left(\left.u_{e x t}\right|_{\Gamma_{e x t}^{\varepsilon}}+\left.u_{i n t}\right|_{\Gamma_{\text {int }}^{\varepsilon}}\right) .
\end{array}\right.
$$

In the same way, for a function $v$, defined over $\Omega$, we define the jump and mean value across an interface $\Gamma$ by:

$$
\left\{\begin{array}{c}
{[v]_{\Gamma}=\left.v_{\text {ext }}\right|_{\Gamma}-\left.v_{\text {int }}\right|_{\Gamma},} \\
\{v\}_{\Gamma}=\frac{1}{2}\left(\left.v_{\text {ext }}\right|_{\Gamma}+\left.v_{\text {int }}\right|_{\Gamma}\right) .
\end{array}\right.
$$

\subsubsection{Second-order model}

The solution of the second-order model satisfies Dirichlet boundary conditions and can be defined independently in the two sub-domains $\Omega_{\text {int }}^{\varepsilon}, \Omega_{\text {ext }}^{\varepsilon}$. Hence, $u^{[1]}=\left(u_{\text {int }}^{[1]}, u_{\text {ext }}^{[1]}\right)$ satisfies

$$
\begin{gathered}
\left\{\begin{array}{rlr}
\sigma_{\mathrm{int}} \Delta u_{\mathrm{int}}^{[1]}=f_{\mathrm{int}} & \text { in } & \Omega_{\mathrm{int}}^{\varepsilon}, \\
u_{\mathrm{int}}^{[1]}=0 & \text { on } & \partial \Omega_{\mathrm{int}}^{\varepsilon} .
\end{array}\right. \\
\left\{\begin{array}{rrr}
\sigma_{\mathrm{ext}} \Delta u_{\mathrm{ext}}^{[1]}=f_{\mathrm{ext}} & \text { in } & \Omega_{\mathrm{ext}}^{\varepsilon}, \\
u_{\mathrm{ext}}^{[1]}=0 & \text { on } & \partial \Omega_{\mathrm{ext}}^{\varepsilon} .
\end{array}\right.
\end{gathered}
$$

\subsubsection{Fourth-order model}

We define a fourth-order approximation $u^{[3]}$, which shall be a much more accurate approximation of $u$ (2) than the second-order model $u^{[1]}$; it solves

$$
\left\{\begin{array}{rlrl}
\sigma_{\text {int }} \Delta u_{\text {int }}^{[3]} & =f_{\text {int }} & \text { in } \quad \Omega_{\text {int }}^{\varepsilon}, \\
\sigma_{\mathrm{ext}} \Delta u_{\mathrm{ext}}^{[3]} & =f_{\mathrm{ext}} & & \text { in } \quad \Omega_{\mathrm{ext}}^{\varepsilon}, \\
{\left[u^{[3]}\right]_{\Gamma^{\varepsilon}}} & =0, & & \\
\sigma_{\text {lay }} \Delta_{\Gamma}\left\{u^{[3]}\right\}_{\Gamma^{\varepsilon}} & =-\varepsilon^{-1}\left[\sigma \partial_{n} u^{[3]}\right]_{\Gamma^{\varepsilon}}-\frac{1}{r_{0}}\left\{\sigma \partial_{n} u^{[3]}\right\}_{\Gamma^{\varepsilon}}, & & \\
u^{[3]} & =0 & & \text { on } \partial \Omega \cap \partial \Omega^{\varepsilon},
\end{array}\right.
$$

where $\Delta_{\Gamma}=\frac{1}{r_{0}^{2}} \partial_{\theta}^{2}+\partial_{z}^{2}$ is the Laplace-Beltrami operator along $\Gamma$ and $\sigma_{\text {lay }}=\widehat{\sigma}_{0} \varepsilon^{-3}$.

\subsection{Asymptotic models with Interface-ITCs}

In this case, the asymptotic method delivers two asymptotic models of first and second orders, respectively. In these models, the exterior and interior problems are uncoupled. 


\subsubsection{First-order model}

The approximate model of order 1 is given by the limit solution $u$ of (2) when $\varepsilon \rightarrow 0$. This solution satisfies homogeneous Dirichlet boundary conditions and can be defined independently in the two sub-domains $\Omega_{\text {int }}, \Omega_{\text {ext }}$. Hence, $u^{[0]}=\left(u_{\mathrm{int}}^{[0]}, u_{\mathrm{ext}}^{[0]}\right)$ satisfies

$$
\begin{aligned}
& \left\{\begin{array}{rrr}
\sigma_{\mathrm{int}} \Delta u_{\mathrm{int}}^{[0]}=f_{\mathrm{int}} & \text { in } & \Omega_{\mathrm{int}}, \\
u_{\mathrm{int}}^{[0]}=0 & \text { on } & \partial \Omega_{\mathrm{int}},
\end{array}\right. \\
& \left\{\begin{array}{rrr}
\sigma_{\mathrm{ext}} \Delta u_{\mathrm{ext}}^{[0]}=f_{\mathrm{ext}} & \text { in } & \Omega_{\mathrm{ext}}, \\
u_{\mathrm{ext}}^{[0]}=0 & \text { on } & \partial \Omega_{\mathrm{ext}} .
\end{array}\right.
\end{aligned}
$$

\subsubsection{Second-order model}

We define a second-order approximation $v^{[1]}$ which satisfies Robin boundary conditions on $\Gamma$ and can be defined independently in the two sub-domains $\Omega_{\text {int }}, \Omega_{\text {ext }}$. Hence, $v^{[1]}=\left(v_{\text {int }}^{[1]}, v_{\text {ext }}^{[1]}\right)$ solves

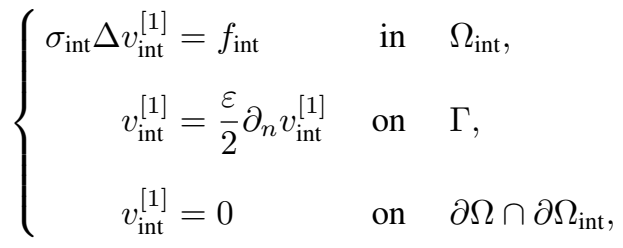

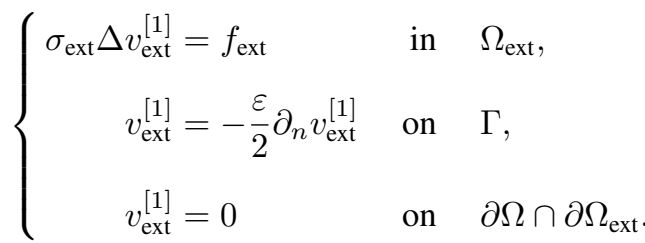

Remark 1. We remark that Model (5) depends on $\sigma_{\text {lay }}=\widehat{\sigma}_{0} \varepsilon^{-3}$, while Models (4), (6), and (7) do not.

We emphasize that the approximations $u^{[0]}(6)$ and $v^{[1]}(7)$ are defined in the thin layer $\Omega_{\text {lay }}^{\varepsilon}$ even if they do not approach a priori the reference solution $u$ in the thin layer, see Th. 9 and Th. 10 (Sec. Appendix B.4.5) for precise error estimates in $\Omega_{\mathrm{int}}^{\varepsilon}$ and in $\Omega_{\mathrm{ext}}^{\varepsilon}$. However, it is possible to give a precise behavior of $u$ in the layer $\Omega_{\text {lay }}^{\varepsilon}$ by using series expansions with profiles $U^{k}$ (see (A.5), Sec. Appendix A.2).

\subsubsection{Stabilized $\delta$-order two model}

It is possible to prove that the Second-order model (7) is not well-posed for all $\varepsilon>0$ and it can be unstable as $\varepsilon$ goes to 0 (see section B.4.2 for more details). To overcome this issue, we introduce the artificial boundaries $\Gamma_{\text {int }}^{\varepsilon \delta}$ and $\Gamma_{\text {ext }}^{\varepsilon \delta}($ see $[9,10])$, which are defined as :

$$
\begin{aligned}
& \Gamma_{\mathrm{int}}^{\varepsilon \delta}=\left\{\left(r_{0}-\delta \varepsilon, \theta, z\right): \delta>0, \theta \in[0,2 \pi), z \in\left(z_{0}, z_{1}\right)\right\}, \\
& \Gamma_{\mathrm{ext}}^{\varepsilon \delta}=\left\{\left(r_{0}+\delta \varepsilon, \theta, z\right): \delta>0, \theta \in[0,2 \pi), z \in\left(z_{0}, z_{1}\right)\right\} .
\end{aligned}
$$

We apply a formal Taylor expansion on variable $r$ to the solution of problem (7), and by neglecting the terms of 
order two or higher in $\varepsilon$, we obtain the following asymptotic model:

$$
\begin{aligned}
& \left\{\begin{array}{rlrl}
\sigma_{\mathrm{int}} \Delta u_{\delta \varepsilon, \mathrm{int}}^{[1]} & =f_{\mathrm{int}} & \text { in } \quad \Omega_{\mathrm{int}}^{\delta \varepsilon}, \\
u_{\delta \varepsilon, \mathrm{int}}^{[1]}=\frac{\varepsilon(1-2 \delta)}{2} \partial_{n} u_{\delta \varepsilon, \mathrm{int}}^{[1]} & \text { on } \quad \Gamma_{\mathrm{int}}^{\delta \varepsilon}, \\
u_{\delta \varepsilon, \mathrm{int}}^{[1]}=0 & \text { on } & \partial \Omega \cap \partial \Omega_{\mathrm{int}}^{\delta \varepsilon},
\end{array}\right. \\
& \left\{\begin{aligned}
\sigma_{\mathrm{ext}} \Delta u_{\delta \varepsilon, \mathrm{ext}}^{[1]}=f_{\mathrm{ext}} & \text { in } \quad \Omega_{\mathrm{ext}}^{\delta \varepsilon}, \\
u_{\delta \varepsilon, \mathrm{ext}}^{[1]}=-\frac{\varepsilon(1-2 \delta)}{2} \partial_{n} u_{\delta \varepsilon, \mathrm{ext}}^{[1]} & \text { on } \quad \Gamma_{\mathrm{ext}}^{\delta \varepsilon}, \\
u_{\delta \varepsilon, \mathrm{ext}}^{[1]}=0 & \text { on } \quad \partial \Omega \cap \partial \Omega_{\mathrm{ext}}^{\delta \varepsilon},
\end{aligned}\right.
\end{aligned}
$$

where domains $\Omega_{\mathrm{int}}^{\delta \varepsilon}$ and $\Omega_{\mathrm{ext}}^{\delta \varepsilon}$ are defined as follows:

$$
\begin{aligned}
& \Omega_{\mathrm{int}}^{\delta \varepsilon}=\left\{(r, \theta, z) \in \mathbb{R}^{3}: 0 \leq r<r_{0}-\delta \varepsilon, 0 \leq \theta<2 \pi, z_{0}<z<z_{1}\right\}, \\
& \Omega_{\mathrm{ext}}^{\delta \varepsilon}=\left\{(r, \theta, z) \in \mathbb{R}^{3}: r_{0}+\delta \varepsilon<r<R_{0}, 0 \leq \theta<2 \pi, z_{0}<z<z_{1}\right\} .
\end{aligned}
$$

With this new formulation, if we select $\delta>\frac{1}{2}$, the stability of the problem is restored. Henceforth, we will refer to this stable model as the Stabilized $\delta$-order two model (see Appendix B.4.4, Theorem 7, for more details).

Remark 2. The stabilized model (9) is a generalized Gap-ITCs model. In particular, when $\delta=\frac{1}{2}$, it corresponds to the second-order model (4).

\section{Numerical results}

\subsection{Convergence results}

Here, we assess the numerical performance of the models defined in the previous sections. For obtaining such numerical results, we employ a finite element code, which allows to solve and analyze both the models with Gap-ITCs and Interface ITCs. First, we provide snapshots of the solutions to the models with Gap-ITCs and we show convergence curves for these models. Then, similar results are presented for the models with Interface-ITCs. Finally, we compare all the analyzed models and remark the strong and weak points of each approach.

The numerical tests are performed in the cylindrical domain $\Omega$ depicted in Figure 1. The subdomains that form $\Omega$ are described in (1), where $R_{0}=2 \mathrm{~m}, r_{0}=1 \mathrm{~m}, z_{0}=0 \mathrm{~m}$, and $z_{1}=1 \mathrm{~m}$. The conductivity $\sigma$ is a piece-wise constant function that takes the following values in each subdomain:

$$
\sigma=\left\{\begin{array}{lll}
\sigma_{\text {int }}=5 \mathrm{~S} / \mathrm{m} & \text { in } & \Omega_{\mathrm{int}}^{\varepsilon}, \\
\sigma_{\text {lay }}=\varepsilon^{-3} \mathrm{~S} / \mathrm{m} & \text { in } & \Omega_{\text {lay }}^{\varepsilon}, \\
\sigma_{\mathrm{ext}}=3 \mathrm{~S} / \mathrm{m} & \text { in } & \Omega_{\mathrm{ext}}^{\varepsilon},
\end{array}\right.
$$

where $\mathrm{S}$ represents Siemens. We select the right hand side function $f$ in the following way:

$$
f=\left\{\begin{array}{lll}
f_{\text {int }}=1 \mathrm{C} & \text { in } & \Omega_{\text {int }}^{\varepsilon}, \\
f_{\text {lay }}=0 \mathrm{C} & \text { in } & \Omega_{\text {lay }}^{\varepsilon}, \\
f_{\text {ext }}=1 \mathrm{C} & \text { in } & \Omega_{\mathrm{ext}}^{\varepsilon} .
\end{array}\right.
$$

where, $\mathrm{C}$ represents Coulomb. We can simplify the $3 \mathrm{D}$ problem into a $2 \mathrm{D}$ problem because the domain, the right hand side function and the parameters are axi-symmetric, and thus, independent of the cylindrical angular variable $\theta$. In this way, we significantly reduce the computational complexity and cost of the numerical simulations. The numerical results contained in this section are obtained by employing piecewise polynomial basis functions of third degree. 


\subsubsection{Gap-ITCs}

This section corresponds to the numerical simulations of the asymptotic models with Gap-ITCs. First, we perform a qualitative comparison by showing some snapshots of the solutions to reference Model (2), and the asymptotic Models (4) and (5). We observe these snapshots in Figure 3. We remark that the fourth-order asymptotic model approximates better the behavior of the reference model. To see this, we consider different values for the thickness $\varepsilon$ of the thin layer, and we calculate the relative error, in $H^{1}$ norm over the domain of the Gap-ITC models, produced by these approximations compared to the reference model. These results are depicted in Figure 4, which clearly shows that the fourth-order model is more accurate than the second-order one. These results also serve the purpose of numerically validating the theoretical convergence rates proved in Appendix B, which indeed coincide.

\subsubsection{Interface-ITCs}

Here we show similar numerical results for the Interface-ITCs. In this case, the asymptotic models (6) and (7) are defined over the domains $\Omega_{\text {int }}$ and $\Omega_{\text {ext }}$, which are described by Equation (3) and Figure 2.

In the same way as for the models with Gap-ITCs, we perform a qualitative comparison between the solution to the reference Model (2) and the asymptotic Models (6) and (7). Figure 5 depicts these solutions. We remark that Panels 5e and 5f show the unstable behavior the theory predicts (See Appendix B). To solve this issue, a new model is proposed in Section 3.2.3. Model (9) makes use of artificial boundaries in order to restore stability. We observe the solution to this model in Figure 6, which shows that instabilities disappear. In addition, we have calculated the relative error, in $H^{1}$ norm over the entire domain without the casing region, produced by the solutions to asymptotic Models (6), (7), and (9), when compared with the solution to the reference Model (2). Figure 7 shows such results for $\delta=0.55$ and different values of the thickness $\varepsilon$ of the thin layer. We observe that while Model (6) converges with its expected theoretical order, Model (7) does not, due to its unstable behavior. On the other hand, Model (9) properly restores stability, and as a result, it converges with its appropriate order.

\subsubsection{Comparison}

This section is devoted to the comparison of the five models we have studied by remarking their advantages and drawbacks. Figure 8 depicts the numerical convergence rates, in $H^{1}$ norm, for all these models. A strong point of the models with Gap-ITCs is that they present higher convergence orders than the models with Interface-ITCs. In this case, we obtain models of order two and four, whereas for the models with Interface-ITCs we obtain convergence orders of one and two.

The main disadvantage of the models with Interface-ITCs is that an unstable Model (7) is delivered, which does not properly converge. However, as it has been shown in the previous sections, this problem can be overcome with the Stabilized version of this model in (9).

An advantage of Interface-ITCs over Gap-ITCs is that the resulting asymptotic models are defined over a domain that is independent of the thickness of the thin layer $\varepsilon$, while the asymptotic models with Gap-ITCs are defined over domains which depend on $\varepsilon$. This fact greatly reduces the complexity of meshing the domain, specially when we deal with curved interfaces separating the different subdomains. We find all these facts summarized in Table 1.

\begin{tabular}{|l|c|c|c|}
\hline Model & Numerical order & Stability & $\varepsilon$-independent domain \\
\hline \hline Gap-ITCs: Order 2 & $\mathbf{2}$ & $\checkmark$ & $\times$ \\
\hline Gap-ITCs: Order 4 & $\mathbf{4}$ & $\checkmark$ & $\mathbf{X}$ \\
\hline Interface-ITCs: Order 1 & $\mathbf{1}$ & $\checkmark$ & $\checkmark$ \\
\hline Interface-ITCs: Order 2 & $\mathbf{1 - 2}$ & $\mathbf{X}$ & $\checkmark$ \\
\hline Stabilized $\delta$-Order 2 & $\mathbf{2}$ & $\checkmark$ & $\mathbf{X}$ \\
\hline
\end{tabular}

Table 1: Comparison of the different derived models. 


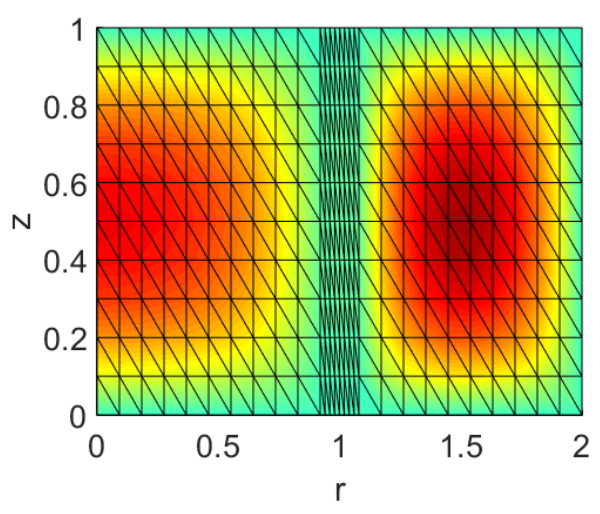

(a) Reference model, $r-z$ view.

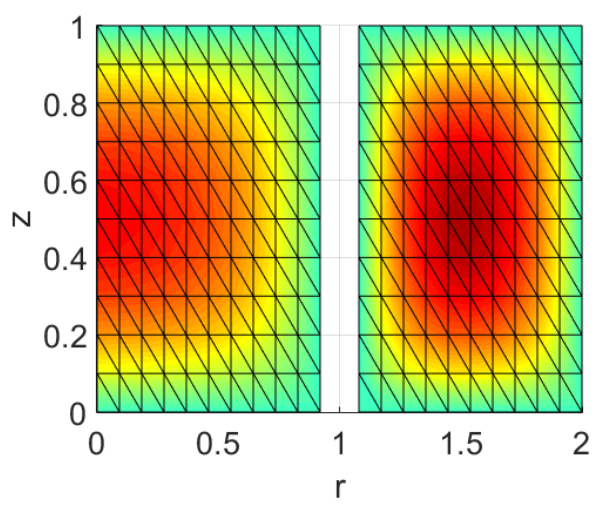

(c) Second-order model, $r-z$ view.

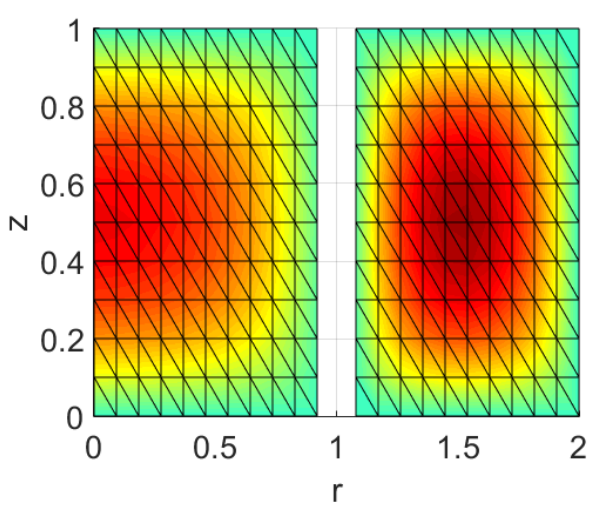

(e) Fourth-order model, $r-z$ view.

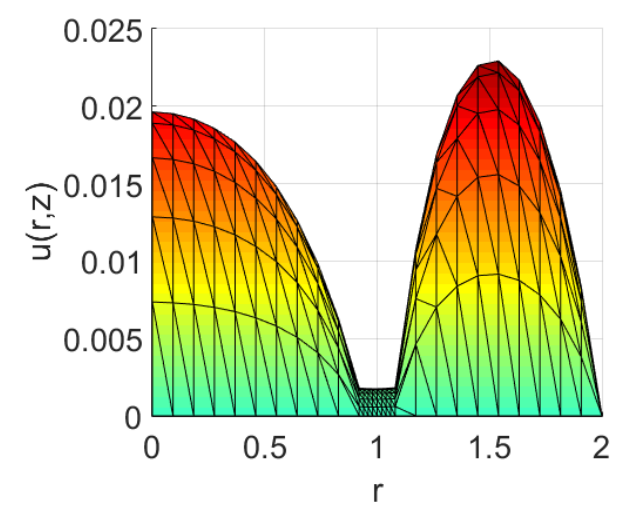

(b) Reference model, $r-u(r, z)$ view.

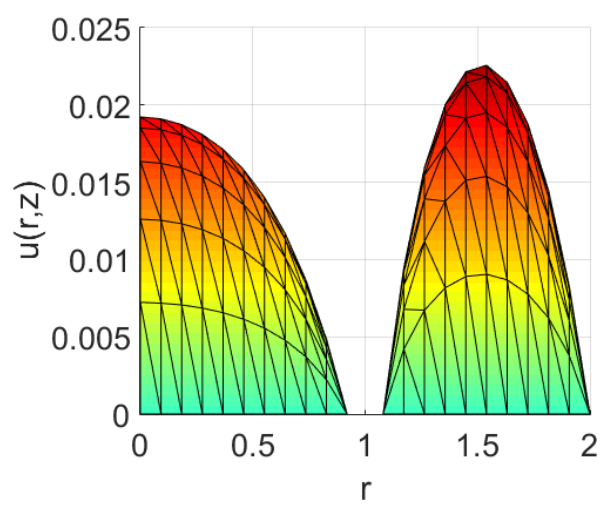

(d) Second-order model, $r-u(r, z)$ view.

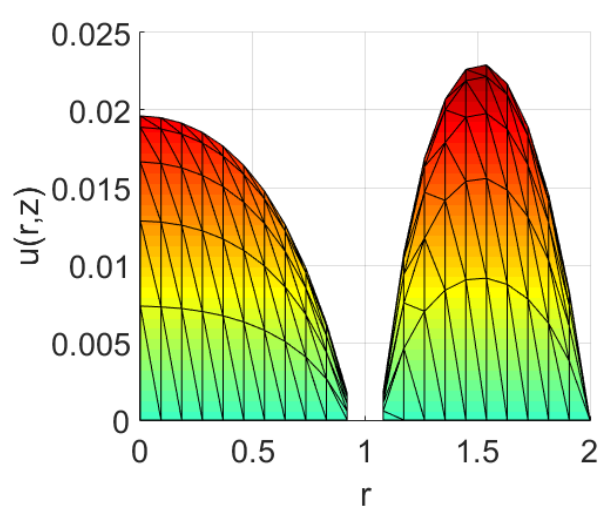

(f) Order 4 model, $r-u(r, z)$ view.

Figure 3: Solution to the reference Problem (2) and the asymptotic models with Gap-ITCs: the second-order Model (4) and the fourth-order Model (5). 


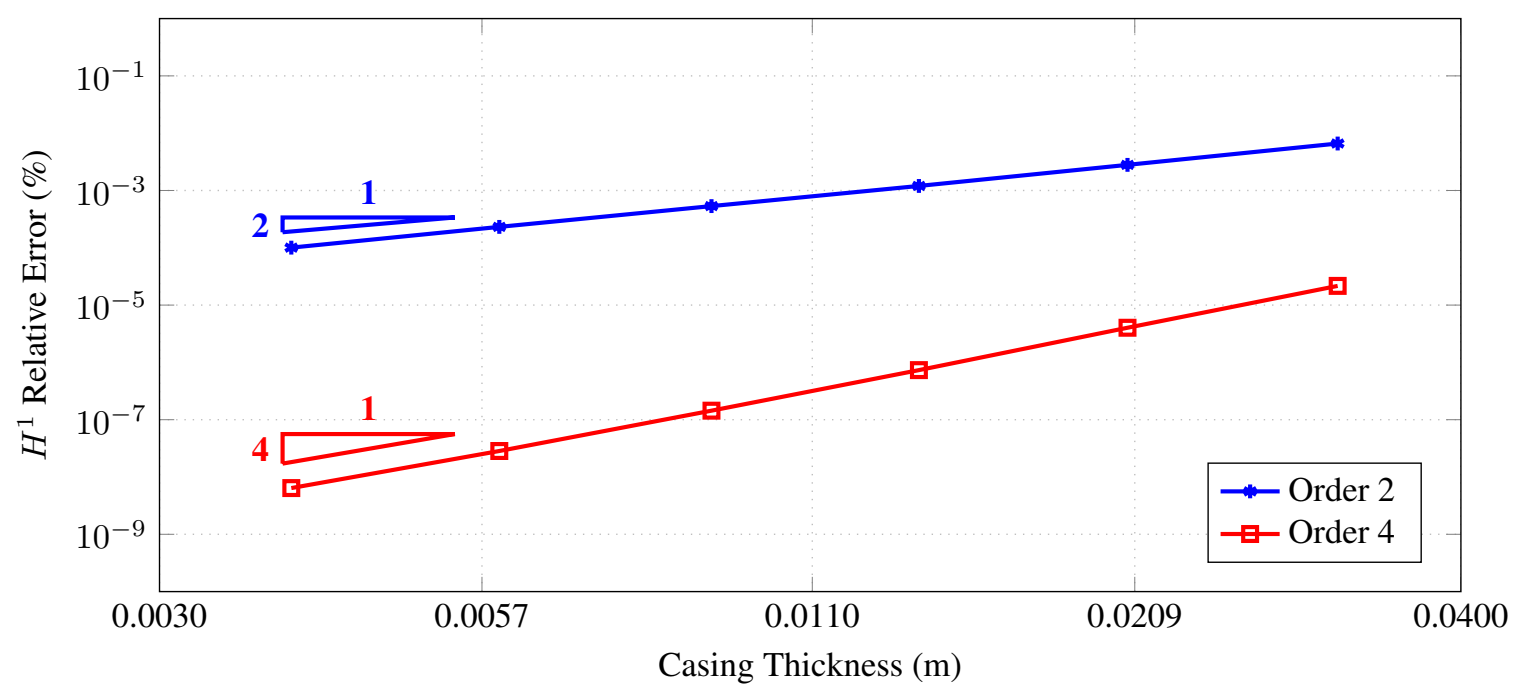

Figure 4: $H^{1}$ relative error produced by the models with Gap-ITCs: the second-order Model (4) and the fourth-order Model (5), for different values of $\varepsilon$.

\subsection{Through-casing simulations}

Here, we show an application of the derived models to the field of resistivity measurements. According to [15], the second derivative of the electric potential along the vertical direction can be employed to determine the resistivity of the rock formations. For this application, we consider the problem set in the domains described by (1), where the parameters that define these domains are selected as follows: $R_{0}=10 \mathrm{~m}, r_{0}=0.16 \mathrm{~m}$, and $z_{1}=-z_{0}=40000 \mathrm{~m}$. The conductivity takes different constant values in every subdomain. Inside the borehole, it takes the value $\sigma_{\text {int }}=1$ $\mathrm{S} / \mathrm{m}$ and inside the metallic casing it takes the value $\sigma_{\text {lay }}=10^{6} \mathrm{~S} / \mathrm{m}$. Inside the domain $\Omega_{\text {ext }}^{\varepsilon}$, which corresponds to the rock formations, several values are considered, which represent different types of fluid-satured rock formations. A transmitter is placed inside the borehole, i.e. in the part of the domain denoted as $\Omega_{\mathrm{int}}^{\varepsilon}$. The right-hand side $f$ represents a ring-source, which is centered at this transmitter. Thus, it takes the following form:

$$
f= \begin{cases}1 \mathrm{C} & \text { at the transmitter, } \\ 0 \mathrm{C} & \text { in the rest of the domain. }\end{cases}
$$

Again, the choice of this right-hand side allows us to reduce the $3 \mathrm{D}$ problem to a $2 \mathrm{D}$ problem, greatly decreasing its complexity. The electric potential should tend to zero when far away from the transmitter. Thus, we consider homogeneous Dirichlet boundary conditions, $u=0$, on the exterior face of the cylinder. On both bases of the cylinder, we consider homogeneous Neumann boundary conditions, $\partial_{n} u=0$. This configuration is depicted in Figure 9.

The objective of these experiments is to measure the electric potential and use these measurements to determine the resistivity of the rock formations surrounding the borehole. For this purpose, three equidistant receivers are placed inside the borehole. These receivers are located $2 \mathrm{~m}$ above the transmitter and there is a $0.16 \mathrm{~m}$ separation between each of them. The three receivers and the transmitter are mounted on the logging instrument, and when this moves along the vertical direction, the distance between the transmitter and receivers remains fixed. Both the transmitter and the receivers are situated $0.05 \mathrm{~m}$ away from the center of the borehole. In these experiments, $\varepsilon=0.01 \mathrm{~m}$.

According to [15], the electric field, when in the presence of a casing, can be divided into a near zone, an intermediate zone and a far zone. In the intermediate zone, the second derivative of the electric potential in the vertical direction can be applied to determine the resistivity of the rock formation. For approximating the second derivative of the potential, we employ the second difference of potential measured at the receivers. Let $\left(r_{1}, z_{r 1}\right),\left(r_{1}, z_{r 2}\right)$, and $\left(r_{1}, z_{r 3}\right)$ be the positions of the first, second, and third receivers, respectively, and let $h$ denote the distance between the receivers. We perform a formal Taylor series expansion on the $z$ variable around the point $\left(r_{1}, z_{r 2}\right)$ and taking into account that $z_{r 1}=z_{r 2}-h$ and $z_{r 3}=z_{r 2}+h$, we obtain the following second difference formula for the second 


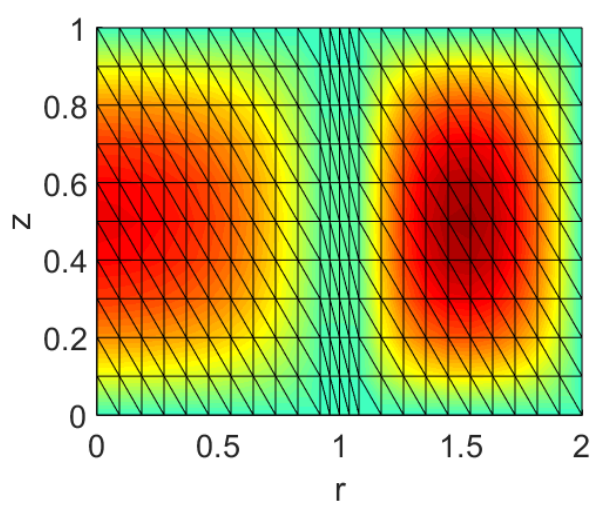

(a) Reference model, $r-z$ view.

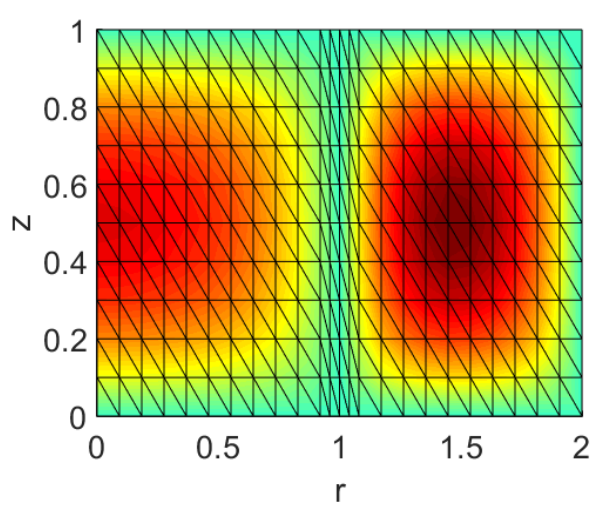

(c) First-order model, $r-z$ view.

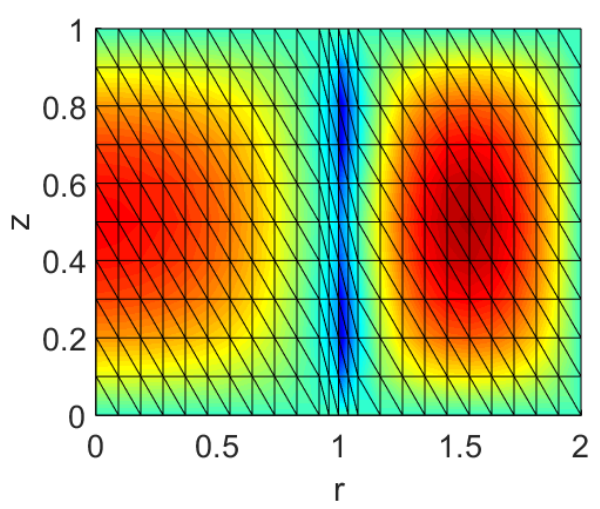

(e) Second-order model, $r-z$ view.

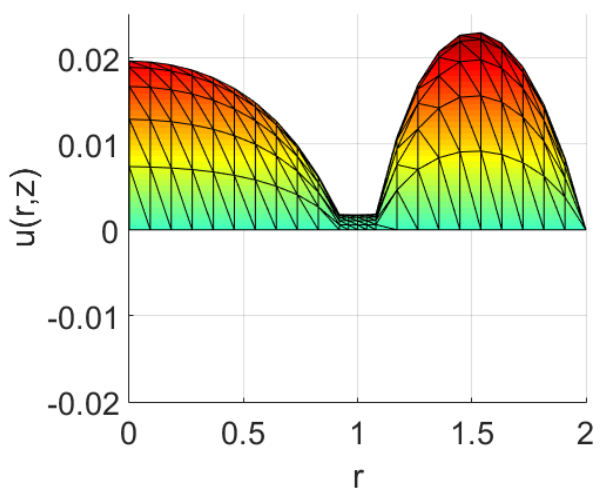

(b) Reference model, $r-u(r, z)$ view.

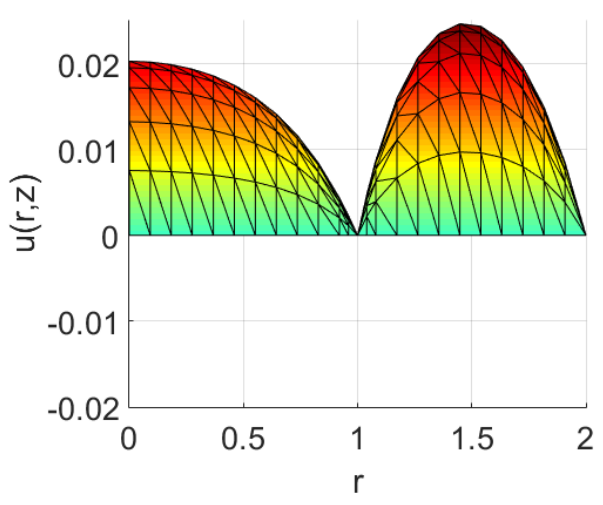

(d) First-order model, $r-u(r, z)$ view.

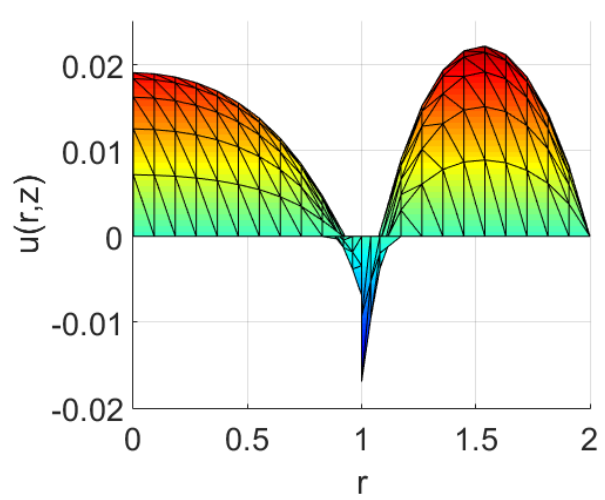

(f) Second-order model, $r-u(r, z)$ view.

Figure 5: Solution to the reference Problem (2) and the asymptotic models with Interface-ITCs: the first-order Model (6) and the second-order Model (7). 


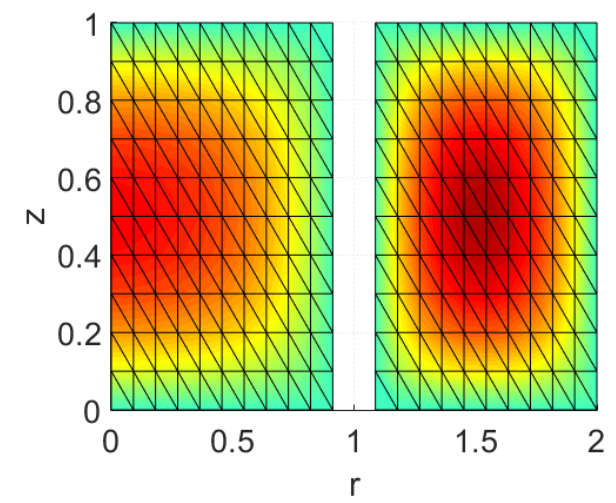

(a) Stabilized $\delta$-order two model, $\mathrm{x}$-y view.

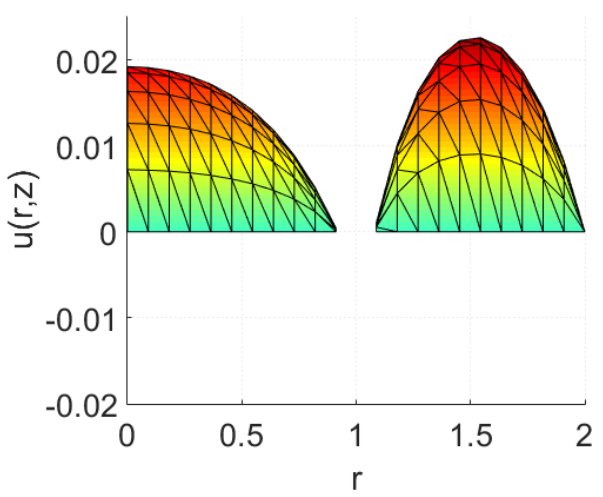

(b) Stabilized $\delta$-order two model, x-z view.

Figure 6: Solution to the Stabilized $\delta$-order two Model (9) with $\delta=0.55$.

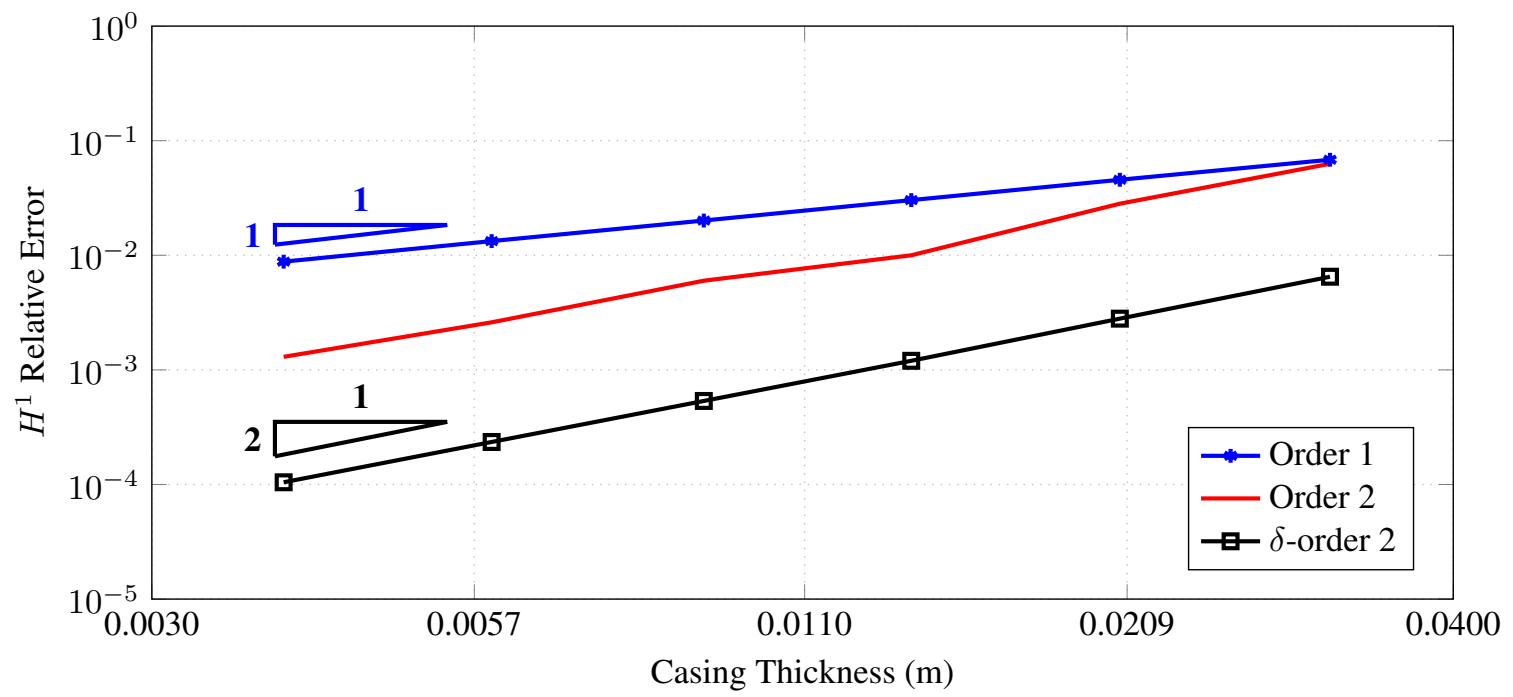

Figure 7: $H^{1}$ relative error of the models with Interface-ITCs: the first-order Model (6), second-order Model (7) and the Stabilized $\delta$-order 2 Model (9), for different values of $\varepsilon$. 


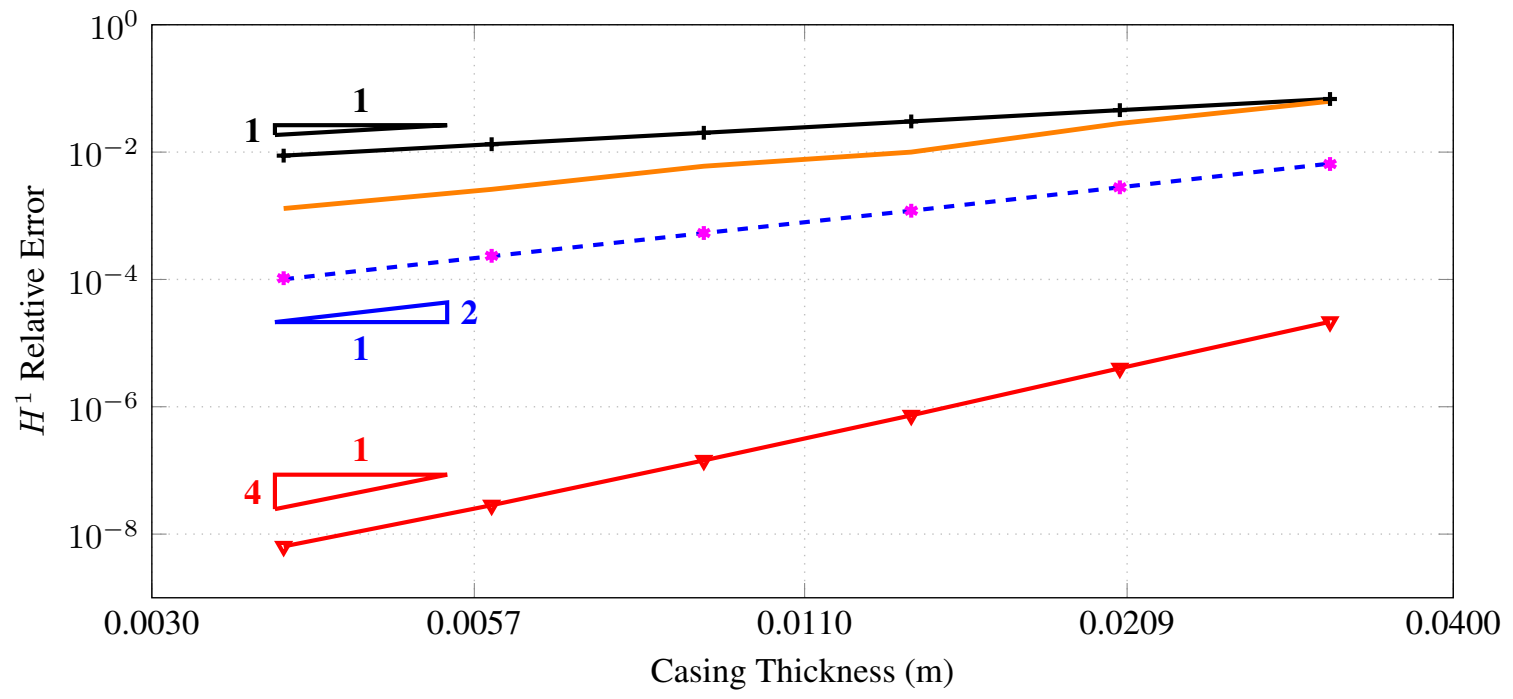

\begin{tabular}{|lllll|}
\hline-- & Gap: order 2 & $\rightarrow$ & Gap: order 4 \\
& Interface: order 2 & $*$ & Stabilized $\delta$-order 2
\end{tabular}

Figure 8: $H^{1}$ relative error of the derived models for different values of $\varepsilon$.

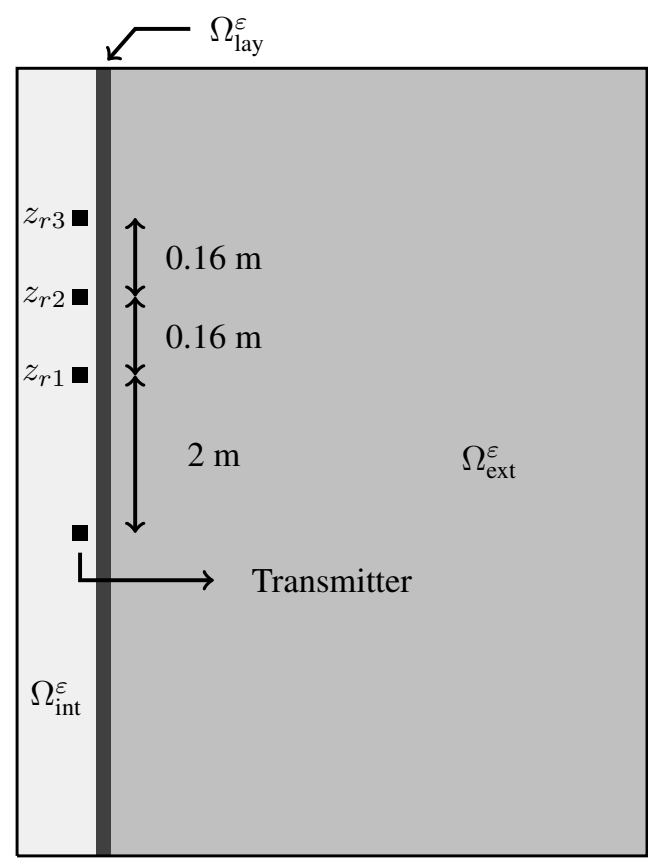

Figure 9: Two-dimensional domain considered for the application. 
derivative of the potential:

$$
\partial_{z}^{2} u\left(r_{1}, z_{r 2}\right)=\frac{u\left(r_{1}, z_{r 3}\right)-2 u\left(r_{1}, z_{r 2}\right)+u\left(r_{1}, z_{r 1}\right)}{h^{2}}+O\left(h^{2}\right) .
$$

If we measure these values at the receivers, we can recover the values of the resistivity in the rock formations, more precisely, the second derivative of the potential should be proportional to the square root of the rock conductivity (see [15]):

$$
\partial_{z}^{2} u\left(r_{1}, z_{r 2}\right) \approx C \sqrt{\sigma_{\mathrm{ext}}}, \quad C>0
$$

\subsubsection{Homogeneous formation}

For the first experiment, we perform several simulations for the electric potential. In each simulation, we consider a different value of the homogeneous resistivity in the domain $\Omega_{\text {ext }}^{\varepsilon}$ varying between $1 \mathrm{Ohm} \cdot \mathrm{m}$ and 10000 $\mathrm{Ohm} \cdot \mathrm{m}$ from one simulation to another. Figure 10 shows the results of measuring the second difference of potential at the receivers for every simulation with different rock resistivities. We compare results for both the reference Model (2) and the fourth-order asymptotic Model (5). The curves are plotted in logarithmic scale and both approximate a slope of -0.5 , which means that the values of the resistivity in the rock formations are properly recovered with both models and that the fourth-order asymptotic model produces a negligible error with respect to the reference model.

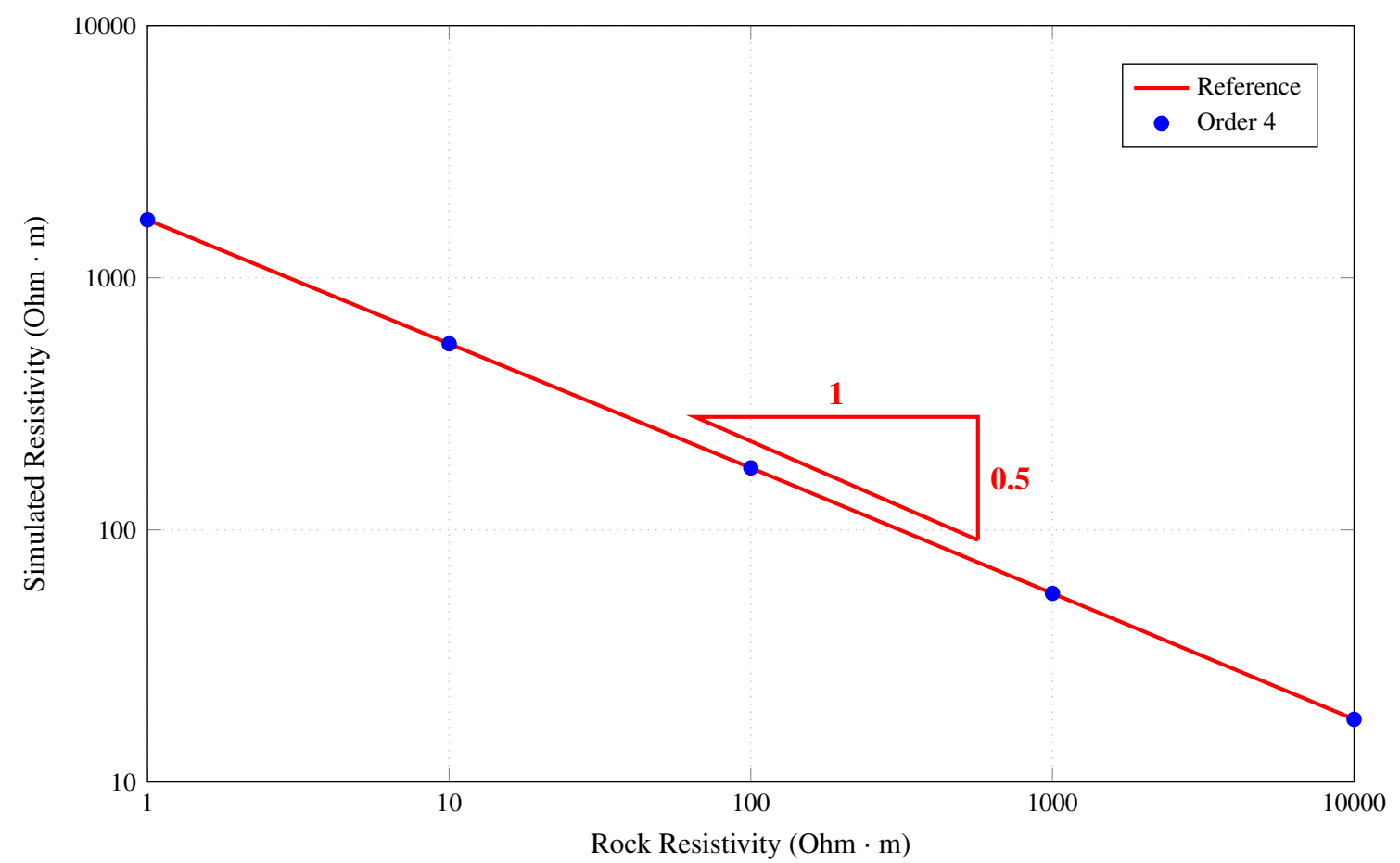

Figure 10: Second difference of potential with different rock resistivities for the reference model (2) and the fourth-order asymptotic model (5).

\subsubsection{Layered formation}

For the second experiment, several layers with different high-contrast conductivity values are considered, as described in Figure 11a. The experiment aims to simulate the apparent conductivities of these rock layers. For that purpose, the instrument is moved along the vertical axis, while the second difference of potential is calculated at the receivers. Figure $11 \mathrm{~b}$ shows the simulated results of such recordings for both the reference Model (2) and the fourth-order asymptotic Model (5). We conclude that the fourth-order asymptotic model produces a negligible error in comparison to the reference model. 


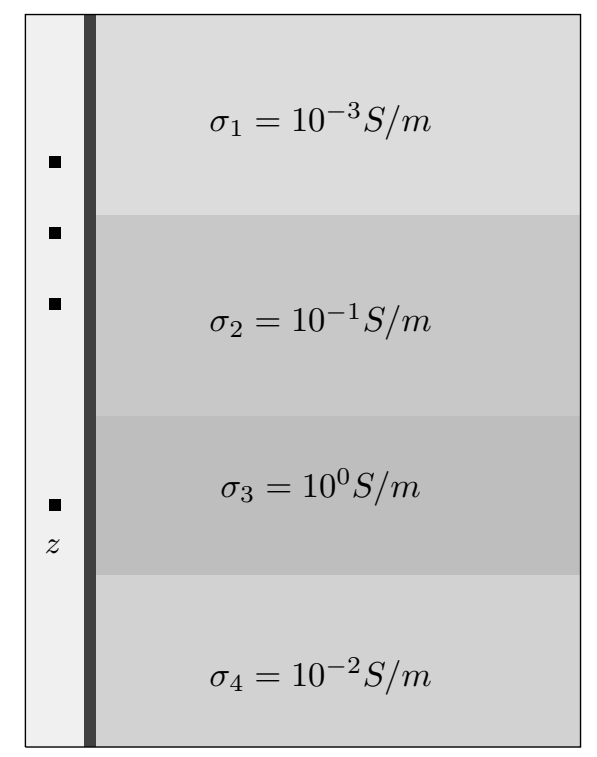

(a) Domain with a formation composed of four layers.

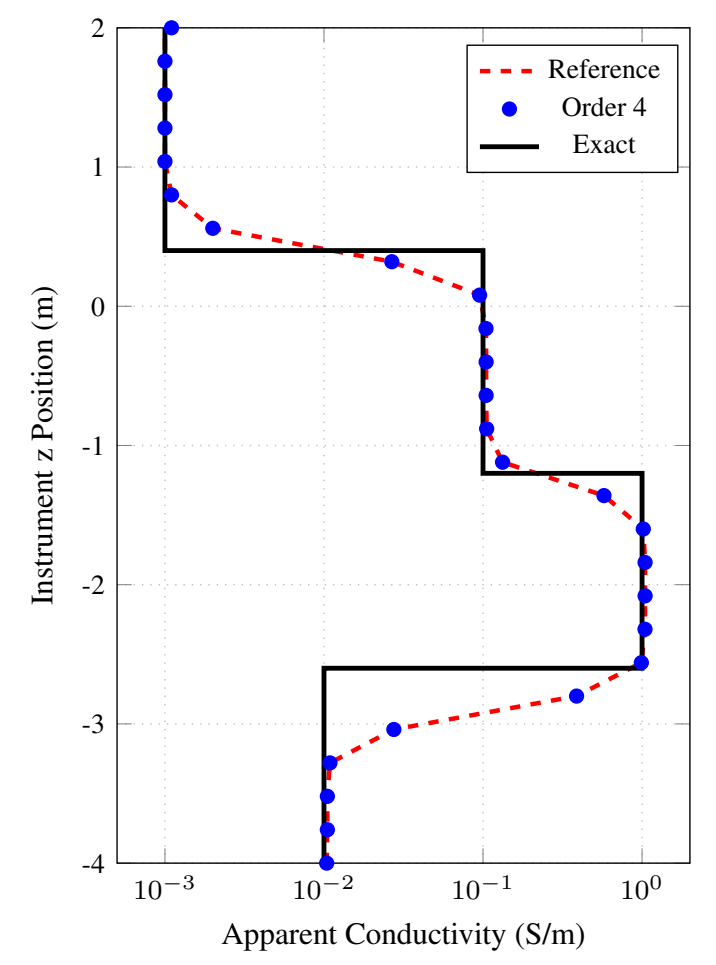

(b) Second difference of potential for different positions of the instrument in a formation composed of four layers for the reference model (2) and the fourth-order asymptotic model (5).

Figure 11: Borehole surrounded by a formation with four layers and second difference of potential measured at the receivers for the reference model (2) and the fourth-order asymptotic model (5). 


\section{Conclusions}

We have derived two families of asymptotic models for simplifying the numerical modeling of through-casing measurements. The first one involves asymptotic models which are defined over the domains $\Omega_{\text {int }}^{\varepsilon}$ and $\Omega_{\text {ext }}^{\varepsilon}$ with GapITCs; these models are of second and fourth orders, respectively. The second ones involves asymptotic models which are defined over a domain that is independent of $\varepsilon$ with interface-ITCs; these models are of first and second orders, respectively. Numerical results confirm the predicted convergence rates.

\section{Appendix A. Derivation of asymptotic models}

\section{Appendix A.1. Scaling}

A key point for the derivation of a multiscale expansion for the solution of Problem (2) consists in performing a scaling along the normal direction to the thin layer. We start by describing domain $\Omega_{\text {lay }}^{\varepsilon}$ in the following way:

$$
\Omega_{\text {lay }}^{\varepsilon}=\left\{\gamma(\theta, z)+\varepsilon R n: \gamma(\theta, z) \in \Gamma, R \in\left(-\frac{1}{2}, \frac{1}{2}\right)\right\}
$$

where $n$ is the normal vector of the interface $\Gamma$ (pointing outwards $\Omega_{\text {int }}$ ), and $\gamma$ is a parametrization of such interface (see Figure 1), which in cylindrical coordinates is given by:

$$
\gamma(\theta, z)=\left(r_{0} \cos \theta, r_{0} \sin \theta, z\right), \text { for all }(\theta, z) \in \Omega^{\theta z} .
$$

Here, $\Omega^{\theta z}$ denotes the domain $[0,2 \pi) \times\left(z_{0}, z_{1}\right)$. This domain geometry induces the following scaling:

$$
r=r_{0}+\varepsilon R \quad \Leftrightarrow \quad R=\varepsilon^{-1}\left(r-r_{0}\right) .
$$

Employing this scaling and performing an expansion in powers of $\varepsilon$, the Laplacian operator takes the following expression inside the thin layer:

$$
\begin{aligned}
\Delta & =\varepsilon^{-2} \partial_{R}^{2}+\sum_{k=0}^{\infty} \varepsilon^{k-1} \frac{(-R)^{k}}{r_{0}^{k+1}} \partial_{R}+\sum_{k=0}^{\infty} \varepsilon^{k}(k+1) \frac{(-R)^{k}}{r_{0}^{k+2}} \partial_{\theta}^{2}+\partial_{z}^{2} \\
& =\varepsilon^{-2} \partial_{R}^{2}+\varepsilon^{-1} \frac{1}{r_{0}} \partial_{R}+\left(-\frac{R}{r_{0}^{2}} \partial_{R}+\frac{1}{r_{0}^{2}} \partial_{\theta}^{2}+\partial_{z}^{2}\right)+\sum_{k=1}^{\infty} \varepsilon^{k} \frac{(-R)^{k}}{r_{0}^{k}}\left((k+1) \partial_{\theta}^{2}-\frac{R}{r_{0}^{2}} \partial_{R}\right) .
\end{aligned}
$$

We assume that we have a solution $u \in H_{0}^{1}(\Omega)$ to (2) and we denote this solution as follows:

$$
u=\left\{\begin{array}{lll}
u_{\text {int }} & \text { in } & \Omega_{\mathrm{int}}^{\varepsilon}, \\
u_{\text {lay }} & \text { in } & \Omega_{\text {lay }}^{\varepsilon}, \\
u_{\text {ext }} & \text { in } & \Omega_{\mathrm{ext}}^{\varepsilon}
\end{array}\right.
$$

Then, employing the scaling (A.1), we define $U$ as the function that satisfies

$$
u_{\text {lay }}(r, \theta, z)=u_{\text {lay }}\left(r_{0}+\varepsilon R, \theta, z\right)=U(R, \theta, z), \quad(R, \theta, z) \in\left(-\frac{1}{2}, \frac{1}{2}\right) \times \Omega^{\theta z} .
$$

Rewriting Equation (2) in each subdomain, it takes the following form outside the thin layer:

$$
\left\{\begin{array}{lll}
\sigma_{\text {int }} \Delta u_{\text {int }}=f_{\text {int }} & \text { in } & \Omega_{\text {int }}^{\varepsilon}, \\
\sigma_{\text {ext }} \Delta u_{\text {ext }}=f_{\text {ext }} & \text { in } & \Omega_{\text {ext }}^{\varepsilon},
\end{array}\right.
$$


and the following form inside the thin layer:

$$
\begin{aligned}
\varepsilon^{-2} \partial_{R}^{2} U+\varepsilon^{-1} \frac{1}{r_{0}} \partial_{R} U+\left(-\frac{R}{r_{0}^{2}} \partial_{R} U\right. & \left.+\frac{1}{r_{0}^{2}} \partial_{\theta}^{2} U+\partial_{z}^{2} U\right) \\
& +\sum_{k=1}^{\infty} \varepsilon^{k} \frac{(-R)^{k}}{r_{0}^{k+2}}\left((k+1) \partial_{\theta}^{2} U-\frac{R}{r_{0}^{2}} \partial_{R} U\right)=0 \quad \text { in }\left(-\frac{1}{2}, \frac{1}{2}\right) \times \Omega^{\theta z},
\end{aligned}
$$

along with the following transmission and boundary conditions:

$$
\left\{\begin{aligned}
u_{\text {int }}\left(r_{0}-\frac{\varepsilon}{2}, \theta, z\right) & =U\left(-\frac{1}{2}, \theta, z\right) & (\theta, z) \in \Omega^{\theta z}, \\
u_{\text {ext }}\left(r_{0}+\frac{\varepsilon}{2}, \theta, z\right) & =U\left(\frac{1}{2}, \theta, z\right) & (\theta, z) \in \Omega^{\theta z}, \\
\sigma_{\text {int }} \partial_{n} u_{\text {int }}\left(r_{0}-\frac{\varepsilon}{2}, \theta, z\right) & =\widehat{\sigma}_{0} \varepsilon^{-4} \partial_{R} U\left(-\frac{1}{2}, \theta, z\right) & (\theta, z) \in \Omega^{\theta z}, \\
\sigma_{\text {ext }} \partial_{n} u_{\text {ext }}\left(r_{0}+\frac{\varepsilon}{2}, \theta, z\right) & =\widehat{\sigma}_{0} \varepsilon^{-4} \partial_{R} U\left(\frac{1}{2}, \theta, z\right) & (\theta, z) \in \Omega^{\theta z}, \\
u & =0 & \text { on } \quad \partial \Omega .
\end{aligned}\right.
$$

Here, $\partial_{n}$ represents the derivative in the direction of the normal vector (inwardly oriented to $\Omega_{\mathrm{ext}}^{\varepsilon}$ on $\Gamma_{\mathrm{ext}}^{\varepsilon}$, and outwardly oriented to $\Omega_{\text {int }}^{\varepsilon}$ on $\Gamma_{\text {int }}^{\varepsilon}$, as shown in Figure 1).

\section{Appendix A.2. Asymptotic expansion}

The following step towards the derivation of Gap-ITCs consists in performing an asymptotic expansion. First, we consider an Ansatz in the form of power series of $\varepsilon$ for the solution to Problems (A.2), (A.3) and (A.4). We look for solutions of the form

$$
\begin{cases}u_{\mathrm{int}}(r, \theta, z) \approx \sum_{k \geq 0} \varepsilon^{k} u_{\mathrm{int}}^{k}(r, \theta, z) & \text { in } \quad \Omega_{\mathrm{int}}^{\varepsilon}, \\ u_{\mathrm{ext}}(r, \theta, z) \approx \sum_{k \geq 0} \varepsilon^{k} u_{\mathrm{ext}}^{k}(r, \theta, z) & \text { in } \quad \Omega_{\mathrm{ext}}^{\varepsilon}, \\ U(R, \theta, z) \approx \sum_{k \geq 0} \varepsilon^{k} U^{k}(R, \theta, z) & \text { in } \quad\left(-\frac{1}{2}, \frac{1}{2}\right) \times \Omega^{\theta z} .\end{cases}
$$

Substituting Expansions (A.5) into Equations (A.2), (A.3), and (A.4), and collecting the terms with the same powers in $\varepsilon$, for every $k \in \mathbb{N}$, we obtain the following set of equations outside the layer:

$$
\left\{\begin{array}{rll}
\sigma_{\mathrm{int}} \Delta u_{\mathrm{int}}^{k}=f_{\mathrm{int}} \delta_{k}^{0} & \text { in } & \Omega_{\mathrm{int}}^{\varepsilon}, \\
\sigma_{\mathrm{ext}} \Delta u_{\mathrm{ext}}^{k}=f_{\mathrm{ext}} \delta_{k}^{0} & \text { in } & \Omega_{\mathrm{ext}}^{\varepsilon},
\end{array}\right.
$$

and the following equations inside the layer:

$$
\begin{aligned}
\partial_{R}^{2} U^{k}+\sum_{l=0}^{k-1} \frac{(-R)^{k-l-1}}{r_{0}^{k-l}} \partial_{R} U^{l}+\sum_{l=0}^{k-2}(k-l-1) \frac{(-R)^{k-l-2}}{r_{0}^{k-l}} \partial_{\theta}^{2} U^{l}+\partial_{z}^{2} U^{k-2}= & 0 \\
& \text { in } \quad\left(-\frac{1}{2}, \frac{1}{2}\right) \times \Omega^{\theta z},
\end{aligned}
$$


along with the following transmission conditions:

$$
\left\{\begin{aligned}
U^{k}\left(-\frac{1}{2}, \theta, z\right) & =u_{\text {int }}^{k}\left(r_{0}-\frac{\varepsilon}{2}, \theta, z\right) & (\theta, z) \in \Omega^{\theta z} \\
U^{k}\left(\frac{1}{2}, \theta, z\right) & =u_{\text {ext }}^{k}\left(r_{0}+\frac{\varepsilon}{2}, \theta, z\right) & (\theta, z) \in \Omega^{\theta z} \\
\partial_{R} U^{k}\left(-\frac{1}{2}, \theta, z\right) & =\frac{\sigma_{\text {int }}}{\widehat{\sigma}_{0}} \partial_{n} u_{\text {int }}^{k-4}\left(r_{0}-\frac{\varepsilon}{2}, \theta, z\right) & (\theta, z) \in \Omega^{\theta z} \\
\partial_{R} U^{k}\left(\frac{1}{2}, \theta, z\right) & =\frac{\sigma_{\text {ext }}}{\widehat{\sigma}_{0}} \partial_{n} u_{\text {ext }}^{k-4}\left(r_{0}+\frac{\varepsilon}{2}, \theta, z\right) & (\theta, z) \in \Omega^{\theta z}
\end{aligned}\right.
$$

and the following boundary conditions:

$$
\left\{\begin{array}{rr}
u^{k}\left(R_{0}, \theta, z\right)=0 & (\theta, z) \in \Omega^{\theta z}, \\
u^{k}\left(r, \theta, z_{0}\right)=u^{k}\left(r, \theta, z_{1}\right)=0 & (r, \theta) \in\left(\left(0, r_{0}-\frac{\varepsilon}{2}\right) \cup\left(r_{0}+\frac{\varepsilon}{2}, R_{0}\right)\right) \\
& \times[0,2 \pi), \\
U^{k}\left(R, \theta, z_{0}\right)=U^{k}\left(R, \theta, z_{1}\right)=0 & (R, \theta) \in\left(-\frac{1}{2}, \frac{1}{2}\right) \times[0,2 \pi) .
\end{array}\right.
$$

For determining the elemental problems satisfied by each of the terms of the expansion, we will also need the following compatibility condition, obtained by applying the fundamental theorem of calculus along with Equations (A.7), (A.8c), and (A.8d) to the function $U^{k}$ :

$$
\begin{aligned}
\int_{\frac{-1}{2}}^{\frac{1}{2}}\left(\partial_{z}^{2} U^{k-2}(R, \theta, z)+\right. & \sum_{l=0}^{k-1} \frac{(-R)^{k-1-l}}{r_{0}^{k-l}} \partial_{R} U^{l}(R, \theta, z) \\
& \left.+\sum_{l=0}^{k-2}(k-l-1) \frac{(-R)^{k-2-l}}{r_{0}^{k-l}} \partial_{\theta}^{2} U^{l}(R, \theta, z)\right) \mathrm{d} R=\frac{-1}{\widehat{\sigma}_{0}}\left[\sigma \partial_{n} u^{k-4}\right]_{\Gamma^{\varepsilon}}(\theta, z)
\end{aligned}
$$

We adopt the convention that the terms with negative indices in Equations (A.6)-(A.10) are equal to zero. Employing Equations (A.6) - (A.10), we obtain the expressions for the elementary problems satisfied outside and inside the layer for any order $k \in \mathbb{N}$. For that purpose, we employ the algorithm described in the following section.

Remark 3. With this approach, the terms of the asymptotic expansion (A.5), a priori still have a dependency with respect to $\varepsilon$ due to the geometry being dependent on $\varepsilon$. It is possible to avoid this dependence by applying a change of variables that delivers an $\varepsilon$-independent geometry and then employing an asymptotic expansion set in $\varepsilon$-independent domains. See [14, Appendix A.8] for more details.

\section{Appendix A.3. Algorithm for the determination of the coefficients}

In this section, we refer the reader to Ref. $[1, \S 6.2 .2]$ where this algorithm is presented with more details.

\section{Appendix A.3.1. Initialization of the algorithm}

Before jumping into the actual steps of the algorithm, we need to determine $U^{0}$ up to an additive function in the variables $\theta$ and $z$, denoted by $\varphi_{0}^{0}$. For that purpose, we consider Equations (A.7), (A.8c), and (A.8d) for building the 
following differential problem in the variable $R$ for $U^{0}$ (variables $\theta$ and $z$ play the role of parameters):

$$
\left\{\begin{aligned}
\partial_{R}^{2} U^{0}(R, \theta, z) & =0 \quad R \in\left(-\frac{1}{2}, \frac{1}{2}\right) \\
\widehat{\sigma}_{0} \partial_{R} U^{0}\left(-\frac{1}{2}, \theta, z\right) & =0 \\
\widehat{\sigma}_{0} \partial_{R} U^{0}\left(\frac{1}{2}, \theta, z\right) & =0
\end{aligned}\right.
$$

From these equations, we conclude that $U^{0}$ has the form $U^{0}(R, \theta, z)=\varphi_{0}^{0}(\theta, z)$, where function $\varphi_{0}^{0}$ has yet to be determined and this is done during the first step of the algorithm. After this preliminary calculation, we move onto determining $U^{k}$ and $u^{k}$ for any order $k \geq 0$.

We assume that the first terms of the expansion (A.5) up to the order $\varepsilon^{k-1}$ have already been calculated and we compute the equations for the $k$-th order. We also assume that at order $k$ we know the form of $U^{k}$ up to an additive function in the variables $\theta$ and $z$, denoted by $\varphi_{0}^{k}$ :

$$
U^{k}(R, \theta, z)=V^{k}(R, \theta, z)+\varphi_{0}^{k}(\theta, z)
$$

where $V^{k}$ has the form

$$
V^{k}(R, \theta, z)=\left\{\begin{array}{rrr}
0 & \text { if } & k=0,1,2,3, \\
\varphi_{k-2}^{k}(\theta, z) R^{k-2}+\varphi_{k-3}^{k}(\theta, z) R^{k-3}+\ldots+\varphi_{1}^{k}(\theta, z) R & \text { if } & k>3 .
\end{array}\right.
$$

The first step consists in obtaining the expression of the function $U^{k+1}$ up to an additive function $\varphi_{0}^{k+1}$. At the second step, we determine the function $\varphi_{0}^{k}$ involved in the expression of $U^{k}$. Finally, we determine $u_{\text {int }}^{k}$ and $u_{\mathrm{ext}}^{k}$ at the third step. For every order $k \geq 0$, the algorithm performs the following steps:

\section{Appendix A.3.2. First step of the algorithm}

We select Equations (A.7), (A.8c), and (A.8d), and we build the following differential problem in the variable $R$ for $U^{k+1}$ (variables $\theta$ and $z$ play the role of parameters):

$$
\left\{\begin{aligned}
\partial_{R}^{2} U^{k+1}(R, \theta, z) & =g^{k+1}(R, \theta, z) \\
\widehat{\sigma}_{0} \partial_{R} U^{k+1}\left(-\frac{1}{2}, \theta, z\right) & =\sigma_{\text {int }} \partial_{n} u_{\text {int }}^{k-3}\left(r_{0}-\frac{\varepsilon}{2}, \theta, z\right) \\
\widehat{\sigma}_{0} \partial_{R} U^{k+1}\left(\frac{1}{2}, \theta, z\right) & =\sigma_{\text {ext }} \partial_{n} u_{\text {ext }}^{k-3}\left(r_{0}+\frac{\varepsilon}{2}, \theta, z\right)
\end{aligned}\right.
$$

where

$$
\begin{aligned}
& g^{k+1}(R, \theta, z)=-\sum_{l=0}^{k} \frac{(-R)^{k-l}}{r_{0}^{k-l+1}} \partial_{R} U^{l}(R, \theta, z)-\sum_{l=0}^{k-1}(k-l) \frac{(-R)^{k-l-1}}{r_{0}^{k-l+1}} \partial_{\theta}^{2} U^{l}(R, \theta, z) \\
&-\partial_{z}^{2} U^{k-1}(R, \theta, z) .
\end{aligned}
$$

There exists a solution $U^{k+1}$ to (A.11) provided the compatibility condition (A.10) is satisfied. We deduce the expression of $U^{k+1}$ as follows

$$
U^{k+1}(R, \theta, z)=V^{k+1}(R, \theta, z)+\varphi_{0}^{k+1}(\theta, z)
$$


where $V^{k+1}$ has the form

$$
V^{k+1}(R, \theta, z)=\left\{\begin{array}{rrr}
0 & \text { if } & k=0,1,2, \\
\varphi_{k-1}^{k+1}(\theta, z) R^{k-1}+\varphi_{k-2}^{k+1}(\theta, z) R^{k-2}+\ldots+\varphi_{1}^{k+1}(\theta, z) R & \text { if } \quad k>2,
\end{array}\right.
$$

see Ref. [1, Prop. 6, §6.2.4]. Function $\varphi_{0}^{k+1}$ is determined at the following order.

\section{Appendix A.3.3. Second step of the algorithm}

We employ the compatibility condition (A.10) (at order $k+2$ ), along with Equation (A.9c) to write the following differential problem in the variables $\theta$ and $z$ for the function $\varphi_{0}^{k}$ (involved in the expression of $U^{k}$ ):

$$
\left\{\begin{aligned}
\partial_{z}^{2} \varphi_{0}^{k}(\theta, z)+\frac{1}{r_{0}^{2}} \partial_{\theta}^{2} \varphi_{0}^{k}(\theta, z) & =h^{k}(\theta, z) & & (\theta, z) \in \Omega^{\theta z}, \\
\varphi_{0}^{k}\left(\theta, z_{0}\right) & =0 & & \theta \in[0,2 \pi), \\
\varphi_{0}^{k}\left(\theta, z_{1}\right) & =0 & & \theta \in[0,2 \pi),
\end{aligned}\right.
$$

where

$$
\begin{aligned}
h^{k}(\theta, z)=-\int_{\frac{-1}{2}}^{\frac{1}{2}} & \left(\partial_{z}^{2} V^{k}(R, \theta, z)+\sum_{l=0}^{k+1} \frac{(-R)^{k+1-l}}{r_{0}^{k+2-l}} \partial_{R} U^{l}(R, \theta, z)\right. \\
& \left.+\sum_{l=0}^{k-1}(k-l+1) \frac{(-R)^{k-l}}{r_{0}^{k+2-l}} \partial_{\theta}^{2} U^{l}(R, \theta, z)+\frac{1}{r_{0}^{2}} \partial_{\theta}^{2} V^{k}(R, \theta, z)\right) \mathrm{d} R-\frac{1}{\widehat{\sigma}_{0}}\left[\sigma \partial_{n} u^{k-2}\right]_{\Gamma^{\varepsilon}}(\theta, z) .
\end{aligned}
$$

By solving this differential equation, we obtain function $\varphi_{0}^{k}$ and thus, the complete expression of $U^{k}$.

\section{Appendix A.3.4. Third step of the algorithm}

We obtain the equations outside the layer by employing Equations (A.6a), (A.6b), (A.8a), (A.8b), (A.9a), and (A.9b). We infer that $u_{\mathrm{int}}^{k}$ and $u_{\mathrm{ext}}^{k}$ are defined independently in the two subdomains $\Omega_{\mathrm{int}}^{\varepsilon}$ and $\Omega_{\mathrm{ext}}^{\varepsilon}$ by equations:

$$
\begin{aligned}
& \left\{\begin{aligned}
\sigma_{\mathrm{int}} \Delta u_{\mathrm{int}}^{k} & =f_{\mathrm{int}} \delta_{k}^{0} & & \text { in } \quad \Omega_{\mathrm{int}}^{\varepsilon}, \\
u_{\mathrm{int}}^{k}\left(r_{0}-\frac{\varepsilon}{2}, \theta, z\right) & =U^{k}\left(-\frac{1}{2}, \theta, z\right), & & \\
u_{\mathrm{int}}^{k} & =0 & & \text { on } \quad \partial \Omega \cap \partial \Omega_{\mathrm{int}}^{\varepsilon},
\end{aligned}\right. \\
& \left\{\begin{aligned}
\sigma_{\mathrm{ext}} \Delta u_{\mathrm{ext}}^{k} & =f_{\mathrm{ext}} \delta_{k}^{0} & & \text { in } \quad \Omega_{\mathrm{ext}}^{\varepsilon}, \\
u_{\mathrm{ext}}^{k}\left(r_{0}+\frac{\varepsilon}{2}, \theta, z\right) & =U^{k}\left(\frac{1}{2}, \theta, z\right), & & \\
u_{\mathrm{ext}}^{k} & =0 & & \text { on } \quad \partial \Omega \cap \partial \Omega_{\mathrm{ext}}^{\varepsilon} .
\end{aligned}\right.
\end{aligned}
$$

Employing this algorithm, we can obtain the expressions for the terms of the expansion for any order $k \geq 0$. The first full steps $(k=0,1,2,3)$ of this algorithm are written in Ref. [1, $\S 6.2 .3]$. With every order, the complexity of the terms increases, and their regularity decreases. Here, we present these expressions up to $k=3$, which provides a good balance between the complexity of the problem and the order of accuracy. 


\section{Appendix A.4. First terms of the asymptotics}

The asymptotic expansion (A.5) has the following form:

$$
\begin{cases}u_{\mathrm{int}}(r, \theta, z)=u_{\mathrm{int}}^{0}(r, \theta, z)+\varepsilon^{2} u_{\mathrm{int}}^{2}(r, \theta, z)+\varepsilon^{3} u_{\mathrm{int}}^{3}(r, \theta, z)+O\left(\varepsilon^{4}\right) & \text { in } \Omega_{\mathrm{int}}^{\varepsilon}, \\ u_{\mathrm{ext}}(r, \theta, z)=u_{\mathrm{ext}}^{0}(r, \theta, z)+\varepsilon^{2} u_{\mathrm{ext}}^{2}(r, \theta, z)+\varepsilon^{3} u_{\mathrm{ext}}^{3}(r, \theta, z)+O\left(\varepsilon^{4}\right) & \text { in } \Omega_{\mathrm{ext}}^{\varepsilon}, \\ U(R, \theta, z)=\varepsilon^{2} \varphi_{0}^{2}(\theta, z)+\varepsilon^{3} \varphi_{0}^{3}(\theta, z)+O\left(\varepsilon^{4}\right) & \text { in }\left(-\frac{1}{2}, \frac{1}{2}\right) \times \Omega^{\theta z},\end{cases}
$$

where functions $u^{k}, k=0,1,2,3$, are defined by Equations (A.13). In particular, we deduce that $u^{0}=\left(u_{\text {int }}^{0}, u_{\text {ext }}^{0}\right)$ solves Problem (4) and $u_{\text {int }}^{1}=u_{\text {ext }}^{1}=0$. The problems satisfied by $u^{k}, k=2,3$, are made explicit in Ref. [1, $\left.\oint 6.2 .3\right]$. For $(R, \theta, z) \in\left(\frac{-1}{2}, \frac{1}{2}\right) \times \Omega^{\theta z}$, functions $U^{k}$ adopt the following form:

$$
U^{k}(R, \theta, z)=\left\{\begin{array}{rl}
0 & k=0,1, \\
\varphi_{0}^{k}(\theta, z) & k=2,3,
\end{array}\right.
$$

where $\varphi_{0}^{2}$ and $\varphi_{0}^{3}$ solve the following equations:

$$
\begin{aligned}
& \left\{\begin{aligned}
\partial_{z}^{2} \varphi_{0}^{2}(\theta, z)+\frac{1}{r_{0}^{2}} \partial_{\theta}^{2} \varphi_{0}^{2}(\theta, z) & =-\frac{1}{\widehat{\sigma}_{0}}\left[\sigma \partial_{r} u^{0}\right]_{\Gamma^{\varepsilon}}(\theta, z) \quad(\theta, z) \in \Omega^{\theta z} \\
\varphi_{0}^{2}\left(\theta, z_{0}\right) & =0 \\
\varphi_{0}^{2}\left(\theta, z_{1}\right) & =0 .
\end{aligned}\right. \\
& \left\{\begin{aligned}
\partial_{z}^{2} \varphi_{0}^{3}(\theta, z)+\frac{1}{r_{0}^{2}} \partial_{\theta}^{2} \varphi_{0}^{3}(\theta, z) & =-\frac{1}{\widehat{\sigma}_{0} r_{0}}\left\{\sigma \partial_{n} u^{0}\right\}_{\Gamma^{\varepsilon}}(\theta, z) \quad(\theta, z) \in \Omega^{\theta z}, \\
\varphi_{0}^{3}\left(\theta, z_{0}\right) & =0, \\
\varphi_{0}^{3}\left(\theta, z_{1}\right) & =0 .
\end{aligned}\right.
\end{aligned}
$$

Appendix A.5. Equivalent models with Gap-ITCs

For deriving equivalent conditions, we truncate the series expansions and we identify a simpler problem satisfied by

$$
u^{(k)}=u^{0}+\varepsilon u^{1}+\ldots+\varepsilon^{k} u^{k} \quad \text { in } \quad \Omega_{\text {int }}^{\varepsilon} \cup \Omega_{\mathrm{ext}}^{\varepsilon}
$$

up to a residual term of order $\varepsilon^{k+1}$. We neglect the residual term of order $\varepsilon^{k+1}$ to obtain an approximate model satisfied by function $u^{[k]}$. We formally derive two approximate models of second and fourth order, respectively.

Appendix A.5.1. Second-order model

We truncate the series from the second term and (using $u^{1}=0$ ) we define $u^{(1)}$ as

$$
u^{(1)}=u^{0}+\varepsilon u^{1}=u^{0} \quad \text { in } \quad \Omega_{\text {int }}^{\varepsilon} \cup \Omega_{\text {ext }}^{\varepsilon} .
$$

From (A.13) $(k=0,1)$, we deduce that $u^{(1)}$ solves the following uncoupled problems:

$$
\begin{aligned}
& \left\{\begin{array}{rll}
\sigma_{\text {int }} \Delta u_{\text {int }}^{(1)}=f_{\text {int }} & \text { in } & \Omega_{\text {int }}^{\varepsilon}, \\
u_{\text {int }}^{(1)}=0 & \text { on } & \partial \Omega_{\mathrm{int}}^{\varepsilon} .
\end{array}\right. \\
& \left\{\begin{array}{rll}
\sigma_{\mathrm{ext}} \Delta u_{\mathrm{ext}}^{(1)}=f_{\mathrm{ext}} & \text { in } & \Omega_{\mathrm{ext}}^{\varepsilon}, \\
u_{\mathrm{ext}}^{(1)}=0 & \text { on } & \partial \Omega_{\mathrm{ext}}^{\varepsilon} .
\end{array}\right.
\end{aligned}
$$


In this case, we have $u^{[1]}=u^{(1)}$, as $u^{(1)}$ does not depend on $\varepsilon$. We infer a second-order model satisfied by $u^{[1]}$, which is the solution to Problem (4).

\section{Appendix A.5.2. Fourth-order model}

We truncate the series at the fourth term and we define $u^{(3)}$ as

$$
u^{(3)}=u^{0}+\varepsilon u^{1}+\varepsilon^{2} u^{2}+\varepsilon^{3} u^{3}=u^{0}+\varepsilon^{2} u^{2}+\varepsilon^{3} u^{3} \quad \text { in } \quad \Omega_{\text {int }}^{\varepsilon} \cup \Omega_{\text {ext }}^{\varepsilon} .
$$

From (A.13) $(k=0,1,2,3)$, we deduce that $u^{(3)}$ satisfies the following equations:

$$
\left\{\begin{aligned}
\sigma_{\mathrm{int}} \Delta u_{\mathrm{int}}^{(3)} & =f_{\mathrm{int}} \quad \text { in } \quad \Omega_{\mathrm{int}}^{\varepsilon}, \\
\sigma_{\mathrm{ext}} \Delta u_{\mathrm{ext}}^{(3)} & =f_{\mathrm{ext}} \quad \text { in } \quad \Omega_{\mathrm{ext}}^{\varepsilon}, \\
{\left[u^{(3)}\right]_{\Gamma^{\varepsilon}} } & =0, \\
\Delta_{\Gamma}\left\{u^{(3)}\right\}_{\Gamma^{\varepsilon}} & =g, \\
u^{(3)} & =0 \quad \text { on } \quad \partial \Omega \cap \partial \Omega^{\varepsilon},
\end{aligned}\right.
$$

where

Rewriting $g$ as

$$
g=-\varepsilon^{2} \frac{1}{\widehat{\sigma}_{0}}\left[\sigma \partial_{n} u^{0}\right]_{\Gamma^{\varepsilon}}-\varepsilon^{3} \frac{1}{\widehat{\sigma}_{0}}\left[\sigma \partial_{n} u^{1}\right]_{\Gamma^{\varepsilon}}-\varepsilon^{3} \frac{1}{\widehat{\sigma}_{0} r_{0}}\left\{\sigma \partial_{n} u^{0}\right\}_{\Gamma^{\varepsilon}}
$$

$$
g=-\varepsilon^{2} \frac{1}{\widehat{\sigma}_{0}}\left[\sigma \partial_{n} u^{(3)}\right]_{\Gamma^{\varepsilon}}-\varepsilon^{3} \frac{1}{\widehat{\sigma}_{0} r_{0}}\left\{\sigma \partial_{n} u^{(3)}\right\}_{\Gamma^{\varepsilon}}+O\left(\varepsilon^{4}\right)
$$

and neglecting the terms of order four or higher in $\varepsilon$, we obtain the fourth-order asymptotic model (5) for $u^{[3]}$.

\section{Appendix A.6. Equivalent models with Interface-ITCs}

Following a similar procedure as for the Gap-ITCs, we derive equivalent models for the Interface-ITCs. The main difference with the previous process consists in the application of a formal Taylor series expansion to the terms of the asymptotic expansion. In here, we extend the domain of definition for these terms, considering thus domain $\Omega$, decomposed into subdomains $\Omega_{\text {int }}$ and $\Omega_{\text {ext }}$ depicted in Figure 2. The terms of the asymptotic expansion are extended up to the interface $\Gamma$ by employing a formal Taylor expansion in the following way:

$$
\begin{aligned}
& u_{\text {int }}^{k}\left(r_{0}-\frac{\varepsilon}{2}, \theta, z\right)=\sum_{j \geq 0} \varepsilon^{j} \frac{(-1)^{j}}{2^{j} j !} \partial_{n}^{j} u_{\text {int }}^{k}\left(r_{0}, \theta, z\right), \\
& u_{\text {ext }}^{k}\left(r_{0}+\frac{\varepsilon}{2}, \theta, z\right)=\sum_{j \geq 0} \varepsilon^{j} \frac{1}{2^{j} j !} \partial_{n}^{j} u_{\text {ext }}^{k}\left(r_{0}, \theta, z\right) .
\end{aligned}
$$

We formally derive two approximate models of first and second order, which are described by (6) and (7), respectively.

In addition, a stabilized model is proposed to deal with the aforementioned stability issues that Model (7) presents. This stabilized model is obtained by considering the artificial boundaries $\Gamma_{\text {int }}^{\varepsilon \delta}$ and $\Gamma_{\text {ext }}^{\varepsilon \delta}$ described in (8) and applying a formal Taylor expansion on the variable $r$ to the solution of problem (7) of the form

$$
\left\{\begin{array}{l}
u_{\mathrm{ext}}^{[2]}\left(r_{0}, \theta, z\right)=u_{\mathrm{ext}}^{[2]}\left(r_{0}+\delta \varepsilon, \theta, z\right)-\delta \varepsilon \partial_{n} u_{\mathrm{ext}}^{[2]}\left(r_{0}+\delta \varepsilon, \theta, z\right)+O\left(\varepsilon^{2}\right), \\
u_{\mathrm{int}}^{[2]}\left(r_{0}, \theta, z\right)=u_{\mathrm{int}}^{[2]}\left(r_{0}-\delta \varepsilon, y\right)+\delta \varepsilon \partial_{n} u_{\mathrm{int}}^{[2]}\left(r_{0}-\delta \varepsilon, \theta, z\right)+O\left(\varepsilon^{2}\right) .
\end{array}\right.
$$

Employing these expressions along with equations (7) and neglecting the terms of order two or higher in $\varepsilon$, we obtain the Stabilized $\delta$-order two asymptotic model, which is described by (9) and defined over domains $\Omega_{\mathrm{int}}^{\delta \varepsilon}$ and $\Omega_{\mathrm{ext}}^{\delta \varepsilon}$. These domains are described in (10). With this new formulation, if we select $\delta>\frac{1}{2}$, the stability of the problem is restored. 


\section{Appendix B. Stability and convergence of the equivalent models}

This section is devoted to the validation of the multiscale expansions performed in Section Appendix A.2. We perform proofs of existence, uniqueness, and uniform estimates for the reference model and then, we prove the stability and convergence of the asymptotic models for both the Gap-ITCs and the Interface-ITCs.

\section{Appendix B.1. The reference model: well-posedness and uniform estimates}

Here we prove that there exists a solution to Problem (2) and that this solution is unique. We consider the following problem:

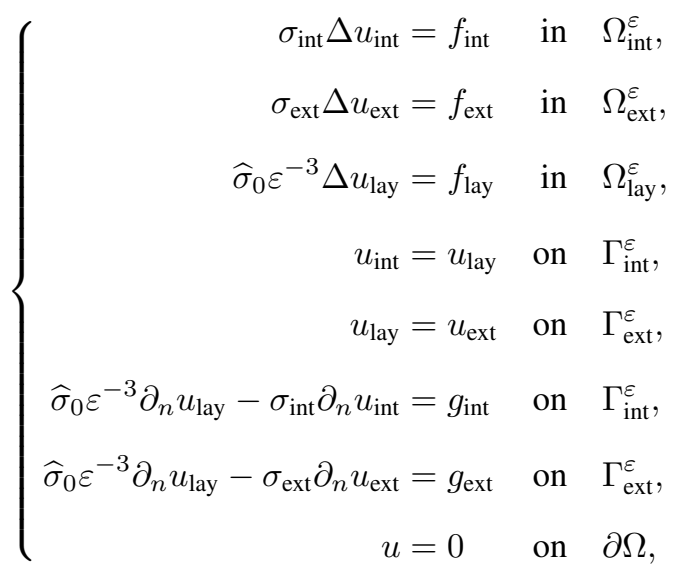

which is defined over the domain depicted in Figure 1 . We remark that constants $\sigma_{\text {int }}, \sigma_{\text {ext }}$, and $\widehat{\sigma}_{0}$ are strictly positive as this fact will play an important role in the following proofs. This problem generalizes Problem (2) by considering the non-zero right-hand side terms $f_{\text {lay }}, g_{\text {int }}$ and $g_{\text {ext }}$. The results obtained for this problem will be useful in later sections, where we prove the convergence of the asymptotic models.

We write the variational formulation of Problem (B.1). Assuming $f \in L^{2}(\Omega), g_{\text {int }} \in L^{2}\left(\Gamma_{\text {int }}^{\varepsilon}\right)$, and $g_{\text {ext }} \in$ $L^{2}\left(\Gamma_{\text {ext }}^{\varepsilon}\right)$, we look for a function $u \in H_{0}^{1}(\Omega)$, such that for all $w \in H_{0}^{1}(\Omega)$,

$$
a(u, w)=l(w)
$$

where

$$
\begin{aligned}
a(u, w) & =\sigma_{\text {int }} \int_{\Omega_{\mathrm{int}}^{\varepsilon}} \nabla u \cdot \nabla w \mathrm{~d} x+\sigma_{\mathrm{ext}} \int_{\Omega_{\text {ext }}^{\varepsilon}} \nabla u \cdot \nabla w \mathrm{~d} x+\widehat{\sigma}_{0} \varepsilon^{-3} \int_{\Omega_{\text {lay }}^{\varepsilon}} \nabla u \cdot \nabla w \mathrm{~d} x, \\
l(w) & =-\int_{\Omega_{\mathrm{int}}^{\varepsilon}} f_{\mathrm{int}} w \mathrm{~d} x-\int_{\Omega_{\mathrm{ext}}^{\varepsilon}} f_{\mathrm{ext}} w \mathrm{~d} x-\int_{\Omega_{\text {lay }}^{\varepsilon}} f_{\text {lay }} w \mathrm{~d} x+\int_{\Gamma_{\mathrm{int}}^{\varepsilon}} g_{\mathrm{int}} w \mathrm{~d} s+\int_{\Gamma_{\text {ext }}^{\varepsilon}} g_{\text {ext }} w \mathrm{~d} s .
\end{aligned}
$$

The following result guarantees the existence and uniqueness of a solution to this problem and presents some uniform estimates for this solution.

Theorem 1. For all $\varepsilon>0$, there exists a unique $u \in H_{0}^{1}(\Omega)$, solution to Problem (B.2), with data $f \in L^{2}(\Omega)$, $g_{\text {int }} \in L^{2}\left(\Gamma_{\text {int }}^{\varepsilon}\right), g_{\text {ext }} \in L^{2}\left(\Gamma_{\text {ext }}^{\varepsilon}\right)$. In addition, there exists $\varepsilon_{0}>0$ and a constant $C>0$, such that for all $\varepsilon \in\left(0, \varepsilon_{0}\right)$,

$$
\|u\|_{1, \Omega} \leq C\left(\|f\|_{0, \Omega}+\left\|g_{i n t}\right\|_{0, \Gamma_{i n t}^{\varepsilon}}+\left\|g_{e x t}\right\|_{0, \Gamma_{e x t}^{\varepsilon}}\right)
$$

Proof. We apply the Poincaré inequality in order to prove the coerciveness of the bilinear form $a$ and place ourselves in the framework of the Lax-Milgram Lemma, which proves the existence and uniqueness of a weak solution. We select

$$
\varepsilon_{0}=\sqrt[3]{\frac{\widehat{\sigma}_{0}}{\min \left(\sigma_{\mathrm{int}}, \sigma_{\mathrm{ext}}\right)}},
$$

which garanties the uniform estimates are satisfied for all $\varepsilon \in\left(0, \varepsilon_{0}\right)$. More details concerning this proof can be found in $[1,14]$. 
Appendix B.2. Convergence of the asymptotic expansion for the reference model

Definition 3. The residue of order $N \in \mathbb{N}$ of the asymptotic expansion (A.5) is defined by removing the first $N$ terms to the solution $u$ of the reference Problem (2):

$$
\begin{cases}r_{\text {int }}^{N}(r, \theta, z)=u_{\text {int }}(r, \theta, z)-\sum_{k=0}^{N} \varepsilon^{k} u_{\text {int }}^{k}(r, \theta, z) & \text { in } \Omega_{\text {int }}^{\varepsilon}, \\ r_{\text {ext }}^{N}(r, \theta, z)=u_{\text {ext }}(r, \theta, z)-\sum_{k=0}^{N} \varepsilon^{k} u_{\text {ext }}^{k}(r, \theta, z) & \text { in } \quad \Omega_{\text {ext }}^{\varepsilon}, \\ r_{\text {lay }}^{N}(r, \theta, z)=u_{\text {lay }}(r, \theta, z)-\sum_{k=0}^{N} \varepsilon^{k} U^{k}\left(\frac{r-r_{0}}{\varepsilon}, \theta, z\right) \text { in } \quad \Omega_{\text {lay }}^{\varepsilon} .\end{cases}
$$

Proposition 1. Let $N \in \mathbb{N}$, the residue $r^{N}$, given by Definition 3, satisfies the following equations:

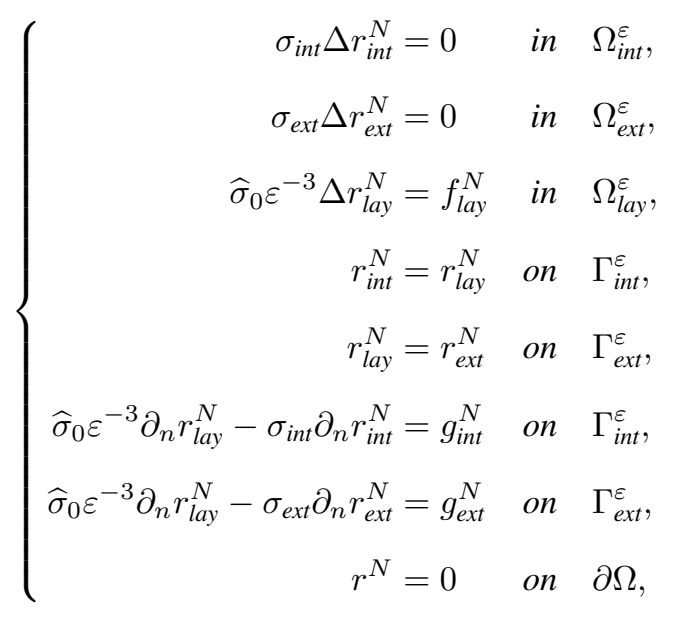

where

$$
\left\|f_{\text {lay }}^{N}\right\|_{L^{2}\left(\Omega_{\text {lay }}^{\varepsilon}\right)}=O\left(\varepsilon^{N-4}\right), \quad\left\|g_{\text {int }}^{N}\right\|_{L^{2}\left(\Gamma_{\text {int }}^{\varepsilon}\right)}=O\left(\varepsilon^{N-3}\right), \quad\left\|g_{\text {ext }}^{N}\right\|_{L^{2}\left(\Gamma_{e x t}^{\varepsilon}\right)}=O\left(\varepsilon^{N-3}\right) .
$$

Proof. We deduce this result by applying Equations (A.6), (A.7), (A.8), (A.9), and (B.1) to the definition of the residue. We refer the reader to Ref. [1, Prop. 3, $\$ 4.1]$ for a detailed proof of this result for a 2D problem, where the right-hand sides $f_{\text {lay }}^{N}, g_{\text {int }}^{N}$, and $g_{\mathrm{ext}}^{N}$ are made explicit.

Theorem 2. Let $N \in \mathbb{N}$. For $\varepsilon \in\left(0, \varepsilon_{0}\right)$ and under the assumptions $f_{\text {lay }}^{N+5} \in L^{2}\left(\Omega_{\text {lay }}^{\varepsilon}\right)$, $g_{\text {int }}^{N+5} \in L^{2}\left(\Gamma_{\text {int }}^{\varepsilon}\right)$, $g_{\text {ext }}^{N+5} \in L^{2}\left(\Gamma_{\text {ext }}^{\varepsilon}\right)$, and $u^{k} \in P H^{1}\left(\Omega^{\varepsilon}\right)$ for $k \leq N+5$, the following estimate holds for the residue of Definition 3 ,

$$
\left\|r_{\text {ext }}^{N}\right\|_{1, \Omega_{\text {ext }}^{\varepsilon}}+\left\|r_{\text {int }}^{N}\right\|_{1, \Omega_{\text {int }}^{\varepsilon}}+\sqrt{\varepsilon}\left\|r_{\text {lay }}^{N}\right\|_{1, \Omega_{\text {lay }}^{\varepsilon}} \leq C \varepsilon^{N+1}
$$

for a positive constant $C>0$ independent of $\varepsilon$.

Proof. The result can be deduced by applying Theorem 1, Proposition 1, and taking into account that $\left\|u_{\text {lay }}^{k}\right\|_{1, \Omega_{\text {lay }}^{\varepsilon}}=$ $O\left(\varepsilon^{-\frac{1}{2}}\right)$. 


\section{Appendix B.3. Validation of the Gap-ITCs}

Appendix B.3.1. Variational formulation of the second-order model

This section is devoted to the derivation of a variational formulation for the second-order asymptotic model we have derived in Section Appendix A.5. Problem (4) is uncoupled into two independent problems. Therefore, the variational formulation will also be uncoupled. We introduce the functional spaces $H_{0}^{1}\left(\Omega_{\mathrm{int}}^{\varepsilon}\right)$ and $H_{0}^{1}\left(\Omega_{\mathrm{ext}}^{\varepsilon}\right)$ as the functional framework. Assuming $f_{\text {int }} \in L^{2}\left(\Omega_{\text {int }}^{\varepsilon}\right)$ and $f_{\text {ext }} \in L^{2}\left(\Omega_{\text {ext }}^{\varepsilon}\right)$, the problem reduces to finding $u_{\text {int }} \in H_{0}^{1}\left(\Omega_{\text {int }}^{\varepsilon}\right)$ such that for all $w_{\text {int }} \in H_{0}^{1}\left(\Omega_{\text {int }}^{\varepsilon}\right)$

$$
-\int_{\Omega_{\text {int }}^{\varepsilon}} f_{\text {int }} w_{\text {int }} \mathrm{d} x=\int_{\Omega_{\text {int }}^{\varepsilon}} \sigma_{\text {int }} \nabla u_{\text {int }} \cdot \nabla w_{\text {int }} \mathrm{d} x,
$$

and finding $u_{\mathrm{ext}} \in H_{0}^{1}\left(\Omega_{\mathrm{ext}}^{\varepsilon}\right)$ such that for all $w_{\mathrm{ext}} \in H_{0}^{1}\left(\Omega_{\mathrm{ext}}^{\varepsilon}\right)$

$$
-\int_{\Omega_{\text {ext }}^{\varepsilon}} f_{\text {ext }} w_{\text {ext }} \mathrm{d} x=\int_{\Omega_{\text {ext }}^{\varepsilon}} \sigma_{\text {ext }} \nabla u_{\text {ext }} \cdot \nabla w_{\text {ext }} \mathrm{d} x .
$$

\section{Appendix B.3.2. Variational formulation of the fourth-order model}

In this section, we derive a variational formulation for the fourth-order asymptotic model we have obtained in Section Appendix A.5. Instead of considering Problem (5) directly, we consider the following problem:

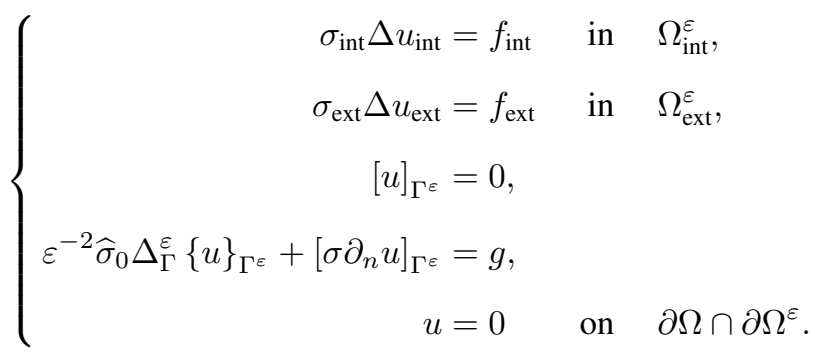

This problem generalizes Problem (5) by including the right-hand side function $g$. We begin by selecting the functional space, which is denoted by $V_{4}$ and defined as follows:

$$
\begin{aligned}
V_{4}=\left\{w: w_{\mathrm{int}} \in H^{1}\left(\Omega_{\mathrm{int}}^{\varepsilon}\right), w_{\mathrm{ext}} \in H^{1}\left(\Omega_{\mathrm{ext}}^{\varepsilon}\right), \nabla_{\Gamma^{\varepsilon}}\{w\} \in L^{2}\left(\Gamma^{\varepsilon}\right),\right. & \\
& \left.\left.w\right|_{\Gamma_{\mathrm{int}}^{\varepsilon}}=\left.w\right|_{\Gamma_{\mathrm{ext}}^{\varepsilon}},\left.w\right|_{\partial \Omega \cap \partial \Omega_{\mathrm{int}}^{\varepsilon}}=0,\left.w\right|_{\partial \Omega \cap \partial \Omega_{\mathrm{ext}}^{\varepsilon}}=0\right\} .
\end{aligned}
$$

Remark 4. Mean values and jumps are defined over the interfaces $\Gamma_{\text {int }}^{\varepsilon}$ and $\Gamma_{\text {ext }}^{\varepsilon}$. As jump and mean values only depend on variable $z, \Gamma^{\varepsilon}$ refers to interval $\left(z_{0}, z_{1}\right)$.

Since $H^{1}\left(\Omega^{\varepsilon}\right)$ is a Hilbert space and $V_{4}$ is a closed subspace of $H^{1}\left(\Omega^{\varepsilon}\right)$, we deduce that functional space $V_{4}$, equipped with the norm

is a Hilbert space.

$$
\|w\|_{V_{4}}=\left(\|w\|_{1, \Omega^{\varepsilon}}^{2}+\left\|\nabla_{\Gamma^{\varepsilon}}\{w\}\right\|_{0, \Gamma^{\varepsilon}}^{2}\right)^{\frac{1}{2}},
$$

For deriving a variational formulation of Problem (B.6), we assume that $f_{\mathrm{int}} \in L^{2}\left(\Omega_{\mathrm{int}}^{\varepsilon}\right)$ and $f_{\mathrm{ext}} \in L^{2}\left(\Omega_{\mathrm{ext}}^{\varepsilon}\right)$. Then, the variational problem reduces to finding $u \in V_{4}$, such that for all $w \in V_{4}$,

$$
a(u, w)=l(w),
$$

where

$$
\begin{gathered}
a(u, w)=\sigma_{\text {int }} \int_{\Omega_{\text {itt }}^{\varepsilon}} \nabla u \cdot \nabla w \mathrm{~d} x+\sigma_{\text {ext }} \int_{\Omega_{\text {ext }}^{\varepsilon}} \nabla u \cdot \nabla w \mathrm{~d} x+\widehat{\sigma}_{0} \varepsilon^{-2} \int_{\Gamma^{\varepsilon}} \nabla_{\Gamma^{\varepsilon}}\{u\}_{\Gamma^{\varepsilon}} \nabla_{\Gamma^{\varepsilon}}\{w\}_{\Gamma^{\varepsilon}} \mathrm{d} s, \\
l(w)=-\int_{\Omega_{\text {int }}^{\varepsilon}} f_{\text {int }} w \mathrm{~d} x-\int_{\Omega_{\text {ext }}^{\varepsilon}} f_{\text {ext }} w \mathrm{~d} x-\int_{\Gamma^{\varepsilon}} g\{w\}_{\Gamma^{\varepsilon}} \mathrm{d} s .
\end{gathered}
$$


Appendix B.3.3. Stability results

We develop an expansion in power series of $\varepsilon$ for Problem (5) in the following form:

$$
\left\{\begin{array}{l}
u_{\mathrm{ext}}^{[3]} \approx \sum_{k \geq 0} \varepsilon^{k} \widehat{u}_{\mathrm{ext}}^{k} \quad \text { in } \quad \Omega_{\mathrm{int}}^{\varepsilon}, \\
u_{\mathrm{int}}^{[3]} \approx \sum_{k \geq 0} \varepsilon^{k} \widehat{u}_{\mathrm{int}}^{k} \quad \text { in } \quad \Omega_{\mathrm{ext}}^{\varepsilon} .
\end{array}\right.
$$

We substitute these expansions in Equations (5) and we collect the terms with the same powers in $\varepsilon$. For every $k \in \mathbb{N}$, we obtain the following set of equations:

$$
\left\{\begin{aligned}
\sigma_{\text {int }} \Delta \widehat{u}_{\text {int }}^{k} & =f_{\text {int }} \delta_{k}^{0} & & \text { in } \quad \Omega_{\text {int }}^{\varepsilon}, \\
\sigma_{\mathrm{ext}} \Delta \widehat{u}_{\mathrm{ext}}^{k} & =f_{\mathrm{ext}} \delta_{k}^{0} & & \text { in } \quad \Omega_{\mathrm{ext}}^{\varepsilon}, \\
{\left[\widehat{u}^{k}\right]_{\Gamma^{\varepsilon}} } & =0, & & \\
-\widehat{\sigma}_{0} \Delta_{\Gamma^{\varepsilon}}\left\{\widehat{u}^{k}\right\}_{\Gamma^{\varepsilon}} & =\left[\sigma \partial_{n} \widehat{u}^{k-2}\right]_{\Gamma^{\varepsilon}}, & & \\
\widehat{u}^{k} & =0 & & \text { on } \partial \Omega \cap \partial \Omega^{\varepsilon} .
\end{aligned}\right.
$$

Definition 4. Given the expansion in power series (B.8) and $N \in \mathbb{N}$, we define the residue $\widehat{r}^{N}$ as

$$
\left\{\begin{array}{l}
\widehat{r}_{i n t}^{N}(x, y)=u_{i n t}^{[3]}(x, y)-\sum_{k=0}^{N} \varepsilon^{k} \widehat{u}_{i n t}^{k}(x, y) \quad \text { in } \quad \Omega_{i n t}^{\varepsilon}, \\
\widehat{r}_{e x t}^{N}(x, y)=u_{\text {ext }}^{[3]}(x, y)-\sum_{k=0}^{N} \varepsilon^{k} \widehat{u}_{\text {ext }}^{k}(x, y) \quad \text { in } \quad \Omega_{\text {ext }}^{\varepsilon} .
\end{array}\right.
$$

Theorem 3. For all $\varepsilon>0$ there exists a unique $u \in V_{4}$ solution to Problem (B.7) with data $f_{\text {int }} \in L^{2}\left(\Omega_{\text {int }}^{\varepsilon}\right)$, $f_{\text {ext }} \in L^{2}\left(\Omega_{\text {ext }}^{\varepsilon}\right)$, and $g \in L^{2}\left(\Gamma^{\varepsilon}\right)$. In addition, there exists $\varepsilon_{0}>0$ and a constant $C>0$, such that for all $\varepsilon \in\left(0, \varepsilon_{0}\right)$,

$$
\|u\|_{V_{4}} \leq C\left(\left\|f_{i n t}\right\|_{0, \Omega_{i n t}^{\varepsilon}}+\left\|f_{e x t}\right\|_{0, \Omega_{e x t}^{\varepsilon}}+\|g\|_{0, \Gamma^{\varepsilon}}\right)
$$

Proof. The proof follows a similar reasoning as for Theorem 1. For proving the uniform estimates, $\varepsilon_{0}=\sqrt{\frac{\widehat{\sigma}_{0}}{\min \left(\sigma_{\text {int }}, \sigma_{\text {ext }}\right)}}$ has to be chosen. More details concerning this proof can be found in [1, 14].

Proposition 2. Let $N \in \mathbb{N}$, the residue $\widehat{r}^{N}$ defined in Definition 4 satisfies the following equations

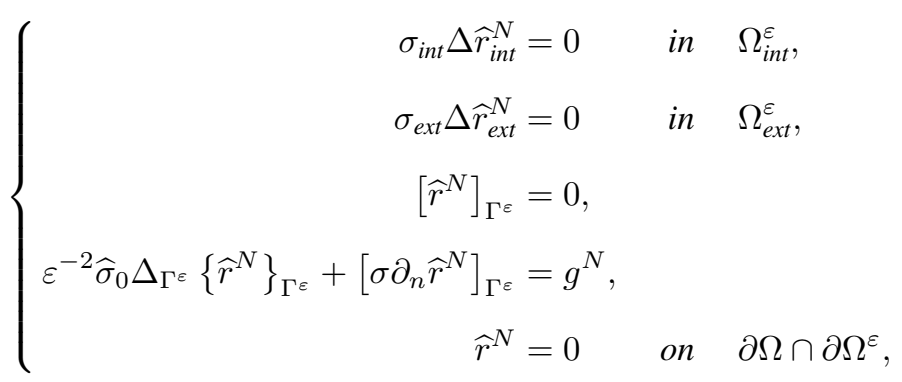

where

$$
g^{N}=\varepsilon^{N+1}\left(-\left[\sigma \partial_{n} \widehat{u}^{N+1}\right]_{\Gamma^{\varepsilon}}-\varepsilon\left[\sigma \partial_{n} \widehat{u}^{N+2}\right]_{\Gamma^{\varepsilon}}\right) .
$$

Proof. We deduce this result by applying Equations (B.9) and (5) to Definition 4. 
Theorem 4. Let $N \in \mathbb{N}$ and $\varepsilon \in\left(0, \varepsilon_{0}\right)$. Under the assumption $g^{N} \in L^{2}\left(\Gamma^{\varepsilon}\right)$, the following estimate holds for the residue $\widehat{r}^{N}$ of Definition 4: there exists a constant $C>0$ independent of $\varepsilon$, such that

$$
\left\|\widehat{r}_{e x t}^{N}\right\|_{1, \Omega_{e x t}^{\varepsilon}}+\left\|\widehat{r}_{i n t}^{N}\right\|_{1, \Omega_{\text {int }}^{\varepsilon}} \leq C \varepsilon^{N+1} \text {. }
$$

Proof. We deduce this result from Theorem 3 and Proposition 2.

\section{Appendix B.3.4. Convergence results}

Theorem 5. Let $u$ be the solution to the reference Problem (2) and let $u^{[1]}$ be the solution to the second-order asymptotic model (4). Under the assumptions of Theorem 2 for $N=1, \varepsilon \in\left(0, \varepsilon_{0}\right)$ and with data $f_{\text {int }} \in L^{2}\left(\Omega_{\text {int }}^{\varepsilon}\right)$ and $f_{\text {ext }} \in L^{2}\left(\Omega_{\text {ext }}^{\varepsilon}\right)$, the following estimate holds: there exists a constant $C>0$ independent of $\varepsilon$, such that

$$
\left\|u_{i n t}-u_{i n t}^{[1]}\right\|_{1, \Omega_{i n t}^{\varepsilon}}+\left\|u_{e x t}-u_{e x t}^{[1]}\right\|_{1, \Omega_{e x t}^{\varepsilon}} \leq C \varepsilon^{2} .
$$

Proof. We deduce this result by applying Theorem 2 while taking into account that $u^{[1]}=u^{(1)}$.

Theorem 6. Let $u$ be the solution to the reference Problem (2) and let $u^{[3]}$ be the solution to the fourth-order asymptotic model (5). Under the assumptions of Theorem 2 and Theorem 4 for $N=3, \varepsilon \in\left(0, \varepsilon_{0}\right)$ and with data $f_{\text {int }} \in L^{2}\left(\Omega_{\text {int }}^{\varepsilon}\right)$ and $f_{\text {ext }} \in L^{2}\left(\Omega_{\text {ext }}^{\varepsilon}\right)$, the following estimate holds: there exists a constant $C>0$ independent of $\varepsilon$, such that

$$
\left\|u_{i n t}-u_{i n t}^{[3]}\right\|_{1, \Omega_{\text {int }}^{\varepsilon}}+\left\|u_{\text {ext }}-u_{\text {ext }}^{[3]}\right\|_{1, \Omega_{e x t}^{\varepsilon}} \leq C \varepsilon^{4}
$$

Proof. We consider expansion (B.8) and Equations (B.9) to deduce that

$$
\widehat{u}^{k} \equiv u^{k} \quad k=0,1,2,3 .
$$

Then, using Theorems 2 and 4 we prove the desired result.

Appendix B.4. Validation of the Interface-ITCs

Appendix B.4.1. First-order model: variational formulation

This section is devoted to the derivation of a variational formulation for the first-order asymptotic model (6), which is uncoupled into two independent problems. Therefore, we write two uncoupled variational formulations. We introduce functional spaces $H_{0}^{1}\left(\Omega_{\text {int }}\right)$ and $H_{0}^{1}\left(\Omega_{\text {ext }}\right)$ as the functional framework. Assuming $f_{\text {int }} \in L^{2}\left(\Omega_{\text {int }}\right)$ and $f_{\text {ext }} \in L^{2}\left(\Omega_{\text {ext }}\right)$, the variational formulations reduce to finding $u_{\text {int }} \in H_{0}^{1}\left(\Omega_{\text {int }}\right)$, such that for all $w_{\text {int }} \in H_{0}^{1}\left(\Omega_{\text {int }}\right)$

$$
-\int_{\Omega_{\text {int }}} f_{\text {int }} w_{\text {int }} \mathrm{d} x=\int_{\Omega_{\text {int }}} \sigma_{\text {int }} \nabla u_{\text {int }} \cdot \nabla w_{\text {int }} \mathrm{d} x
$$

and finding $u_{\mathrm{ext}} \in H_{0}^{1}\left(\Omega_{\mathrm{ext}}\right)$, such that for all $w_{\mathrm{ext}} \in H_{0}^{1}\left(\Omega_{\mathrm{ext}}\right)$

$$
-\int_{\Omega_{\mathrm{ext}}} f_{\mathrm{ext}} w_{\mathrm{ext}} \mathrm{d} x=\int_{\Omega_{\mathrm{ext}}} \sigma_{\mathrm{ext}} \nabla u_{\mathrm{ext}} \cdot \nabla w_{\mathrm{ext}} \mathrm{d} x .
$$

Appendix B.4.2. Second-order model: variational formulation

In this section, we derive a variational formulation for the second-order asymptotic model (7) we have derived in Section 3.2.3. We introduce the functional spaces $V_{\text {int }}$ and $V_{\text {ext }}$, which are defined as follows:

$$
\begin{aligned}
& V_{\mathrm{int}}=\left\{w \in H^{1}\left(\Omega_{\mathrm{int}}\right):\left.w\right|_{\partial \Omega \cap \partial \Omega_{\mathrm{int}}}=0\right\}, \\
& V_{\mathrm{ext}}=\left\{w \in H^{1}\left(\Omega_{\mathrm{ext}}\right):\left.w\right|_{\partial \Omega \cap \partial \Omega_{\mathrm{ext}}}=0\right\} .
\end{aligned}
$$

Assuming $f_{\text {int }} \in L^{2}\left(\Omega_{\text {int }}\right)$ and $f_{\text {ext }} \in L^{2}\left(\Omega_{\text {ext }}\right)$, the variational formulations consist in finding $u_{\text {int }} \in V_{\text {int }}$, such that for all $w_{\text {int }} \in V_{\text {int }}$

$$
-\int_{\Omega_{\text {int }}} f_{\text {int }} w_{\text {int }} \mathrm{d} x=\int_{\Omega_{\text {int }}} \sigma_{\text {int }} \nabla u_{\text {int }} \cdot \nabla w_{\text {int }} \mathrm{d} x-\int_{\Gamma} \frac{2 \sigma_{\text {int }}}{\varepsilon} u_{\text {int }} w_{\text {int }} \mathrm{d} s
$$


and finding $u_{\text {ext }} \in V_{\text {ext }}$, such that for all $w_{\text {ext }} \in V_{\text {ext }}$

$$
-\int_{\Omega_{\mathrm{ext}}} f_{\mathrm{ext}} w_{\mathrm{ext}} \mathrm{d} x=\int_{\Omega_{\mathrm{ext}}} \sigma_{\mathrm{ext}} \nabla u_{\mathrm{ext}} \cdot \nabla w_{\mathrm{ext}} \mathrm{d} x-\int_{\Gamma} \frac{2 \sigma_{\text {ext }}}{\varepsilon} u_{\mathrm{ext}} w_{\mathrm{ext}} \mathrm{d} s .
$$

Observing these variational formulations, we notice that the bilinear forms are not coercive anymore, contrary to the first-order model. This is due to the terms

$$
-\int_{\Gamma} \frac{2 \sigma_{\text {int }}}{\varepsilon} u_{\text {int }} w_{\text {int }} \mathrm{d} s \quad \text { and } \quad-\int_{\Gamma} \frac{2 \sigma_{\text {ext }}}{\varepsilon} u_{\text {ext }} w_{\text {ext }} \mathrm{d} s .
$$

For $\varepsilon$ given, the self-adjoint operator associated with the bilinear form can be split into a coercive operator and a compact operator. As a consequence, the problem is not well-posed (the problem might have non trivial solutions or there can be no solution) for the values of $\varepsilon$ such that $2 / \varepsilon$ coincides with the Steklov eigenvalues $\left(\lambda_{n}\right)_{n \in \mathbb{N}}($ this sequence goes to $+\infty$ ) of the mixed Steklov-Dirichlet problems

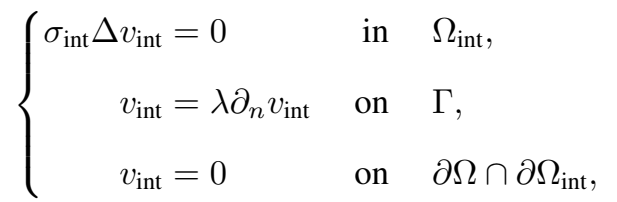

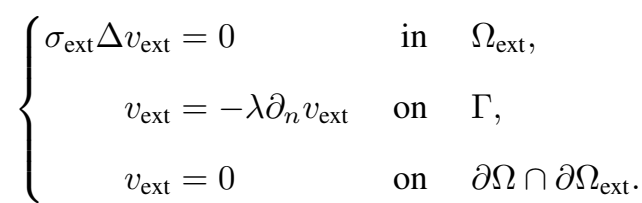

The second-order model set in $\Omega_{\text {int }}$ (resp. in $\Omega_{\text {ext }}$ ) still admits a unique solution when $\lambda_{n} \neq \frac{2}{\varepsilon}$ for all $n \in \mathbb{N}$. However, the norm of the inverse of the operator might be very large since the distance to the spectrum might decrease as $\varepsilon$ goes to 0 , which could cause numerical instabilities.

\section{Appendix B.4.3. Stabilized $\delta$-order two model: variational formulation}

In this section, we derive a variational formulation for the Stabilized $\delta$-order two model (9) we have derived in Section 3.2.3. Instead of directly considering Problem (9), we will consider the following problem:

$$
\begin{aligned}
& \left\{\begin{aligned}
\sigma_{\text {int }} \Delta u_{\text {int }}=f_{\text {int }} & \text { in } \quad \Omega_{\text {int }}^{\delta}, \\
u_{\text {int }}-\frac{\varepsilon(1-2 \delta)}{2} \partial_{n} u_{\text {int }}=g_{\text {int }} & \text { on } \quad \Gamma_{\text {int }}^{\delta}, \\
u_{\text {int }}=0 & \text { on } \quad \partial \Omega \cap \partial \Omega_{\mathrm{int}}^{\delta},
\end{aligned}\right. \\
& \left\{\begin{array}{rll}
\sigma_{\mathrm{ext}} \Delta u_{\mathrm{ext}}=f_{\mathrm{ext}} & \text { in } \quad \Omega_{\mathrm{ext}}^{\delta}, \\
u_{\mathrm{ext}}+\frac{\varepsilon(1-2 \delta)}{2} \partial_{n} u_{\mathrm{ext}}=g_{\mathrm{ext}} & \text { on } \quad \Gamma_{\mathrm{ext}}^{\delta}, \\
u_{\mathrm{ext}}=0 & \text { on } \quad \partial \Omega \cap \partial \Omega_{\mathrm{ext}}^{\delta} .
\end{array}\right.
\end{aligned}
$$

This problem is similar to Problem (9) and it generalizes it by including the right-hand side functions $g_{1}$ and $g_{2}$. We introduce functional spaces $V_{\text {int }}^{\delta}$ and $V_{\text {ext }}^{\delta}$, which are defined as follows:

$$
\begin{gathered}
V_{\mathrm{int}}^{\delta}=\left\{w \in H^{1}\left(\Omega_{\mathrm{int}}^{\delta}\right):\left.w\right|_{\partial \Omega \cap \partial \Omega_{\mathrm{int}}^{\delta}}=0\right\}, \\
V_{\mathrm{ext}}^{\delta}=\left\{w \in H^{1}\left(\Omega_{\mathrm{ext}}^{\delta}\right):\left.w\right|_{\partial \Omega \cap \partial \Omega_{\mathrm{ext}}^{\delta}}=0\right\} .
\end{gathered}
$$


Assuming $f_{\text {int }} \in L^{2}\left(\Omega_{\text {int }}^{\delta}\right)$ and $f_{\text {ext }} \in L^{2}\left(\Omega_{\text {ext }}^{\delta}\right)$, the variational formulations reduce to finding $u_{\text {int }} \in V_{\text {int }}^{\delta}$, such that for all $w_{\text {int }} \in V_{\text {int }}^{\delta}$

$$
a_{\text {int }}\left(u_{\text {int }}, w_{\text {int }}\right)=l_{\text {int }}\left(w_{\text {int }}\right),
$$

and finding $u_{\mathrm{ext}} \in V_{\mathrm{ext}}^{\delta}$, such that for all $w_{\mathrm{ext}} \in V_{\mathrm{ext}}^{\delta}$

$$
a_{\text {ext }}\left(u_{\text {ext }}, w_{\text {ext }}\right)=l_{\text {ext }}\left(w_{\text {ext }}\right)
$$

where

and

$$
\begin{aligned}
& a_{\text {int }}\left(u_{\text {int }}, w_{\text {int }}\right)=\int_{\Omega_{\text {int }}^{\delta}} \sigma_{\text {int }} \nabla u_{\text {int }} \cdot \nabla w_{\text {int }} \mathrm{d} x-\int_{\Gamma_{\text {int }}^{\delta}} \frac{2 \sigma_{\text {int }}}{\varepsilon(1-2 \delta)} u_{\text {int }} w_{\text {int }} \mathrm{d} s, \\
& a_{\text {ext }}\left(u_{\text {ext }}, w_{\text {ext }}\right)=\int_{\Omega_{\text {ext }}^{\delta}} \sigma_{\text {ext }} \nabla u_{\text {ext }} \cdot \nabla w_{\text {ext }} \mathrm{d} x-\int_{\Gamma_{\text {ext }}^{\delta}} \frac{2 \sigma_{\text {ext }}}{\varepsilon(1-2 \delta)} u_{\text {ext }} w_{\text {ext }} \mathrm{d} s,
\end{aligned}
$$

$$
\begin{aligned}
& l_{\text {int }}\left(w_{\text {int }}\right)=-\int_{\Omega_{\text {int }}^{\delta}} f_{\text {int }} w_{\text {int }} \mathrm{d} x-\int_{\Gamma_{\text {int }}^{\delta}} \frac{2 \sigma_{\text {int }}}{\varepsilon(1-2 \delta)} g_{\text {int }} w_{\text {int }} \mathrm{d} s, \\
& l_{\text {ext }}\left(w_{\text {ext }}\right)=-\int_{\Omega_{\text {ext }}^{\delta}} f_{\text {ext }} w_{\text {ext }} \mathrm{d} x-\int_{\Gamma_{\text {ext }}^{\delta}} \frac{2 \sigma_{\text {ext }}}{\varepsilon(1-2 \delta)} g_{\text {ext }} w_{\text {ext }} \mathrm{d} s .
\end{aligned}
$$

With these variational formulations, we observe that if we select $\delta>\frac{1}{2}$ we enforce the coerciveness of the corresponding bilinear form.

\section{Appendix B.4.4. Stability results}

We first develop an expansion in power series of $\varepsilon$ for Problem (9) in the form

$$
\begin{cases}u_{\delta, \mathrm{ext}}^{[1]} \approx \sum_{k \geq 0} \varepsilon^{k} \widehat{u}_{\delta, \mathrm{ext}}^{k} & \text { in } \quad \Omega_{\mathrm{int}}^{\delta}, \\ u_{\delta, \mathrm{int}}^{[1]} \approx \sum_{k \geq 0} \varepsilon^{k} \widehat{u}_{\delta, \mathrm{int}}^{k} & \text { in } \Omega_{\mathrm{ext}}^{\delta} .\end{cases}
$$

We substitute these series into Equations (9) and we collect the terms with the same powers in $\varepsilon$. For every $k \in \mathbb{N}$, we obtain the following set of equations:

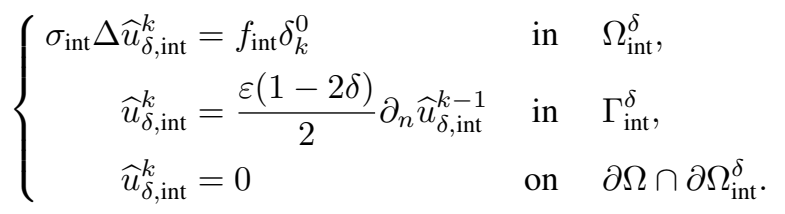

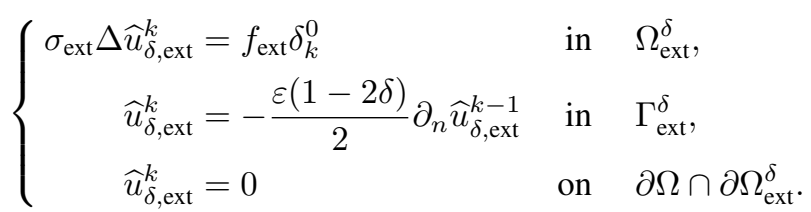

Definition 5. Given the expansion in power series (B.15) and $N \in \mathbb{N}$, we define the residue $\widehat{r}_{\delta}^{N}$ as

$$
\left\{\begin{array}{l}
\widehat{r}_{\delta, \text { int }}^{N}(x, y)=u_{\delta, \text { int }}^{[3]}(x, y)-\sum_{k=0}^{N} \varepsilon^{k} \widehat{u}_{\delta, \text { int }}^{k}(x, y), \\
\widehat{r}_{\delta, \text { ext }}^{N}(x, y)=u_{\delta, \text { ext }}^{[3]}(x, y)-\sum_{k=0}^{N} \varepsilon^{k} \widehat{u}_{\delta, \text { ext }}^{k}(x, y),
\end{array}\right.
$$


Theorem 7. For all $\varepsilon>0$ and $\delta>\frac{1}{2}$, there exists a unique $u=\left(u_{\text {int }}, u_{\text {ext }}\right)$ where $u_{\text {int }} \in V_{\text {int }}^{\delta}$ and $u_{\text {ext }} \in V_{\text {ext }}^{\delta}$ are solutions to (B.13) and (B.14) respectively with data $f_{\text {int }} \in L^{2}\left(\Omega_{\text {int }}^{\delta}\right), f_{\text {ext }} \in L^{2}\left(\Omega_{\text {ext }}^{\delta}\right), g_{\text {int }} \in L^{2}\left(\Gamma_{\text {int }}^{\delta}\right), g_{\text {ext }} \in L^{2}\left(\Gamma_{\text {ext }}^{\delta}\right)$. In addition, there exists $\varepsilon_{0}$ and a constant $C>0$ such that for all $\varepsilon \in\left(0, \varepsilon_{0}\right)$

$$
\|u\|_{1, \Omega^{\delta}} \leq \varepsilon^{-1} C\left(\left\|f_{\text {int }}\right\|_{0, \Omega_{\text {int }}^{\delta}}+\left\|f_{\text {ext }}\right\|_{0, \Omega_{\text {ext }}^{\delta}}+\left\|g_{\text {int }}\right\|_{0, \Gamma_{\text {int }}^{\delta}}+\left\|g_{\text {ext }}\right\|_{0, \Gamma_{\text {ext }}^{\delta}}\right) .
$$

Proof. The proof follows a similar reasoning to the one explained in Theorems 1 and 3 . The proof of uniform estimates requires the choice of $\varepsilon_{0}=\frac{2 k \sigma_{\text {int }}}{2 \delta-1}$, where the constant $k>0$ represents a trace constant. More details concerning this proof can be found in $[1,14]$.

Proposition 3. Let $N \in \mathbb{N}$. The residue $\widehat{r}_{\delta}^{N}$ of Definition 5 satisfies the following equations:

$$
\begin{gathered}
\left\{\begin{aligned}
\sigma_{\text {int }} \Delta \widehat{r}_{\delta, \text { int }}^{N}=0 & \text { in } \quad \Omega_{\text {int }}^{\delta}, \\
\widehat{r}_{\delta, \text { int }}^{N}-\frac{\varepsilon(1-2 \delta)}{2} \widehat{r}_{\delta, \text { int }}^{N}=g_{\text {int }}^{N} & \text { on } \quad \Gamma_{\text {int }}^{\delta}, \\
\widehat{r}_{\delta, \text { int }}^{N}=0 & \text { on } \quad \partial \Omega \cap \partial \Omega_{\text {int }}^{\delta} .
\end{aligned}\right. \\
\left\{\begin{array}{rr}
\sigma_{\text {int }} \Delta \widehat{r}_{\delta, \text { ext }}^{N}=0 & \text { in } \quad \Omega_{\text {ext }}^{\delta}, \\
\widehat{r}_{\delta, \text { ext }}^{N}+\frac{\varepsilon(1-2 \delta)}{2} \widehat{r}_{\delta, \text { ext }}^{N}=g_{\text {ext }}^{N} & \text { on } \quad \Gamma_{\text {ext }}^{\delta}, \\
\widehat{r}_{\delta, \text { ext }}^{N}=0 & \text { on } \quad \partial \Omega \cap \partial \Omega_{\text {ext }}^{\delta},
\end{array}\right.
\end{gathered}
$$

where

$$
\begin{aligned}
& g_{i n t}^{N}=\varepsilon^{N+1} \frac{1-2 \delta}{2} \partial_{n} u_{i n t}^{N}, \\
& g_{\text {ext }}^{N}=\varepsilon^{N+1} \frac{1-2 \delta}{2} \partial_{n} u_{e x t}^{N} .
\end{aligned}
$$

Proof. We deduce this result by applying Equations (B.16) and (9) to the definition of the residue.

Theorem 8. Let $N \in \mathbb{N}$. For $\varepsilon \in\left(0, \varepsilon_{0}\right)$ and under the assumptions $g_{i n t}^{N} \in L^{2}\left(\Gamma_{i n t}^{\delta}\right), g_{\text {ext }}^{N} \in L^{2}\left(\Gamma_{\text {ext }}^{\delta}\right), g_{\text {int }}^{N+1} \in L^{2}\left(\Gamma_{\text {int }}^{\delta}\right)$, $g_{\text {ext }}^{N+1} \in L^{2}\left(\Gamma_{\text {ext }}^{\delta}\right)$, and $u^{k} \in H^{1}\left(\Omega^{\varepsilon}\right)$ for $k \leq N+1$, there exists a constant $C>0$, independent of $\varepsilon$, for which the following estimate holds for the residue $\widehat{r}_{\delta}^{N}$ of Definition 5:

$$
\left\|\widehat{r}_{\delta, \text { ext }}^{N}\right\|_{1, \Omega_{\text {ext }}^{\delta}}+\left\|\widehat{r}_{\delta, i n t}^{N}\right\|_{1, \Omega_{\text {int }}^{\delta}} \leq C \varepsilon^{N+1}
$$

Proof. We deduce this result from Theorem 7 and Proposition 3.

Appendix B.4.5. Convergence results

Theorem 9. Let $u$ be the solution to reference Problem (2) and let $u^{[0]}$ the solution to the first-order asymptotic model (6). Under the assumptions of Theorem 2 for $N=0$ and $\varepsilon \in\left(0, \varepsilon_{0}\right)$, with the data $f_{\text {int }} \in L^{2}\left(\Omega_{\text {int }}\right)$ and $f_{\text {ext }} \in L^{2}\left(\Omega_{\text {ext }}\right)$, the following estimate holds: there exists a constant $C>0$ independent of $\varepsilon$, such that

$$
\left\|u_{i n t}-u_{i n t}^{[0]}\right\|_{1, \Omega_{i n t}^{\varepsilon}}+\left\|u_{e x t}-u_{e x t}^{[0]}\right\|_{1, \Omega_{e x t}^{\varepsilon}} \leq C \varepsilon .
$$

Proof. We deduce this result from Theorem 2 while taking into account that $u^{[0]}=u^{(0)}$. 
Theorem 10. Let $u$ be the solution to reference Problem (2) and let $u_{\delta}^{[1]}$ be the solution to the Stabilized $\delta$-order two model (9). Under the assumptions of Theorem 2 and Theorem 8 for $N=1$ and $\varepsilon \in\left(0, \varepsilon_{0}\right)$, with data $f_{\text {int }} \in L^{2}\left(\Omega_{\text {int }}\right)$ and $f_{\text {ext }} \in L^{2}\left(\Omega_{\text {ext }}\right)$, the following estimate holds: there exists a constant $C>0$ independent from $\varepsilon$, such that

$$
\left\|u_{i n t}-u_{\delta, \text { int }}^{[1]}\right\|_{1, \Omega_{\text {int }}^{\delta}}+\left\|u_{e x t}-u_{\delta, \text { ext }}^{[1]}\right\|_{1, \Omega_{e x t}^{\delta}} \leq C \varepsilon^{2} .
$$

Proof. We consider Expansion (B.15) and Equations (B.16) along with Theorems 7, 2 and 8 to prove the desired result.

\section{Acknowledgments}

The authors have received funding from the European Union's Horizon 2020 research and innovation programme under the Marie Sklodowska-Curie grant agreement No 644602 and 777778. David Pardo has also received funding the Projects of the Spanish Ministry of Economy and Competitiveness with reference MTM2016-76329-R (AEI/FEDER, EU), and MTM2016-81697-ERC/AEI, the BCAM "Severo Ochoa" accreditation of excellence SEV2013-0323, and the Basque Government through the BERC 2014-2017 program, and the Consolidated Research Group Grant IT649-13 on "Mathematical Modeling, Simulation, and Industrial Applications (M2SI)".

\section{References}

[1] H. Barucq, A. Erdozain, and V. Péron. Impedance Transmission Conditions for the Electric Potential across a Highly Conductive Casing. Research Report RR-8998, Inria Bordeaux Sud-Ouest, December 2016.

[2] A.-L. Bessoud, F. Krasucki, and M. Serpilli. Asymptotic analysis of shell-like inclusions with high rigidity. Journal of Elasticity, 103(2):153$172,2011$.

[3] M. Bonnet, A. Burel, M. Duruflé, and P. Joly. Effective transmission conditions for thin-layer transmission problems in elastodynamics. The case of a planar layer model. ESAIM: Mathematical Modelling and Numerical Analysis, 50:43-75, 2016.

[4] Q. Chen, D. Pardo, H. Li, and F. Wang. Compensation effect analysis in DIE method for through-casing measuring formation resistivity. Journal of Applied Geophysics, 74(4):287-293, 2011.

[5] Q. Chen, D. Pardo, H. Li, and F. Wang. New post-processing method for interpretation of through casing resistivity (TCR) measurements. Journal of Applied Geophysics, 74(1):19-25, 2011.

[6] S. Chun, H. Haddar, and J. S. Hesthaven. High-order accurate thin layer approximations for time-domain electromagnetics. Part II: transmission layers. Journal of Computational and Applied Mathematics, 234(8):2587-2608, 2010.

[7] S. Chun and J. S. Hesthaven. High-order accurate thin layer approximations for time-domain electromagnetics. Part I: General metal backed coatings. Journal of computational and applied mathematics, 231(2):598-611, 2009.

[8] M. Dauge. Neumann and mixed problems on curvilinear polyhedra. Integral Equations Operator Theory, 15(2):227-261, 1992.

[9] B. Delourme. Modèles et asymptotiques des interfaces fines et périodiques en électromagnétisme. PhD thesis, Université Pierre et Marie Curie-Paris VI, 2010.

[10] B. Delourme, H. Haddar, and P. Joly. Approximate models for wave propagation across thin periodic interfaces. Journal de mathématiques pures et appliquées, 98(1):28-71, 2012.

[11] J. Diaz and V. Péron. Equivalent Robin boundary conditions for acoustic and elastic media. Math. Models Methods Appl. Sci., 26(8):1531$1566,2016$.

[12] M. Duruflé, V. Péron, and C. Poignard. Time-harmonic maxwell equations in biological cells. the differential form formalism to treat the thin layer. Confluentes Mathematici, 3(02):325-357, 2011.

[13] M. Duruflé, V. Péron, and C. Poignard. Thin layer models for electromagnetism. Communications in Computational Physics, 16:213-238, 2014.

[14] A. Erdozain. Fast inversion of 3D Borehole Resistivity Measurements using Model Reduction Techniques based on 1D Semi-Analytical Solutions. PhD thesis, Université de Pau et des Pays de l'Adour, Universidad del País Vasco - Euskal Herriko Unibertsitatea, 2016.

[15] A. Kaufman. The electrical field in a borehole with a casing. Geophysics, 55(1):29-38, 1990.

[16] A. Kaufman and E. Wightman. A transmission-line model for electrical logging through casing. Geophysics, 58(12):1739-1747, 1993.

[17] D. Pardo, L. Demkowicz, C. Torres-Verdín, and C Michler. PML enhanced with a self-adaptive goal-oriented hp-finite element method: Simulation of through-casing borehole resistivity measurements. SIAM Journal on Scientific Computing, 30(6):2948-2964, 2008.

[18] D. Pardo, L. Demkowicz, C. Torres-Verdín, and L. Tabarovsky. A goal-oriented hp-adaptive finite element method with electromagnetic applications. Part I: electrostatics. International Journal for Numerical Methods in Engineering, 65(8):1269-1309, 2006.

[19] D. Pardo, C. Torres-Verdín, and L. Demkowicz. Simulation of multifrequency borehole resistivity measurements through metal casing using a goal-oriented hp finite-element method. Geoscience and Remote Sensing, IEEE Transactions on, 44(8):2125-2134, 2006.

[20] D. Pardo, C. Torres-Verdín, and L. Demkowicz. Feasibility study for 2D frequency-dependent electromagnetic sensing through casing. Geophysics, 72(3):F111-F118, 2007.

[21] D. Pardo, C. Torres-Verdin, and M. Paszynski. Simulations of 3D DC borehole resistivity measurements with a goal-oriented hp finite-element method. Part II: through-casing resistivity instruments. Computational Geosciences, 12(1):83-89, 2008. 
[22] D. Pardo, C. Torres-Verdín, and Z. Zhang. Sensitivity study of borehole-to-surface and crosswell electromagnetic measurements acquired with energized steel casing to water displacement in hydrocarbon-bearing layers. Geophysics, 73(6):F261-F268, 2008

[23] V. Péron. Mathematical modeling of electromagnetic phenomena in high contrast media. Theses, Université Rennes 1, September 2009. date de rédaction: avril 2009.

[24] V. Péron. Equivalent boundary conditions for an elasto-acoustic problem set in a domain with a thin layer. ESAIM: Mathematical Modelling and Numerical Analysis, 48(5):1431-1449, 2014.

[25] V. Péron. Asymptotic Models for Acoustic, Elastic and Electromagnetic Media. Corner and Edge Asymptotics for Elliptic Systems. Habilitation à diriger des recherches, Université de Pau et des Pays de l'Adour, December 2017.

[26] V. Péron, K. Schmidt, and M. Duruflé. Equivalent transmission conditions for the time-harmonic Maxwell equations in 3D for a medium with a highly conductive thin sheet. SIAM Journal on Applied Mathematics, 76(3):1031-1052, 2016.

[27] R. Perrussel and C. Poignard. Asymptotic expansion of steady-state potential in a high contrast medium with a thin resistive layer. Applied Mathematics and Computation, 221:48-65, 2013.

[28] K. Schmidt and A. Chernov. A unified analysis of transmission conditions for thin conducting sheets in the time-harmonic eddy current model. SIAM Journal on Applied Mathematics, 73(6):1980-2003, 2013.

[29] K. Schmidt and S. Tordeux. High order transmission conditions for thin conductive sheets in magneto-quasistatics. ESAIM: Mathematical Modelling and Numerical Analysis, 45(06):1115-1140, 2011. 